\title{
Genome-wide identification, gene cloning, subcellular location and expression analysis of SPL gene family in $P$. granatum $\mathrm{L}$
}

Bianbian Li ${ }^{1,2}$, Yujie Zhao ${ }^{1,2}$, Sha Wang ${ }^{1,2}$, Xinhui Zhang ${ }^{1,2}$, Yongwei Wang ${ }^{1,2}$, Yu Shen ${ }^{1,2}$ and Zhaohe Yuan ${ }^{1,2^{*}}$

\begin{abstract}
Backgrounds: Pomegranate is an excellent tree species with nutritional, medicinal, ornamental and ecological values. Studies have confirmed that SPL factors play an important role in floral transition and flower development.

Results: Used bioinformatics methods, 15 SPL (SQUAMOSA promoter-binding protein-like) genes were identified and analyzed from the 'Taishanhong' pomegranate (P. granatum L.) genome. Phylogenetic analysis showed that PgSPLs were divided into six subfamilies (G1 G6). PgSPL promoter sequences contained multiple cis-acting elements associated with abiotic stress or hormonal response. Based on the transcriptome data, expression profiles of different tissues and different developmental stages showed that PgSPL genes had distinct temporal and spatial expression characteristics. The expression analysis of miR156 in small RNA sequencing results showed that miR156 negatively regulated the expression of target genes. qRT-PCR analysis showed that the expression levels of PgSPL2, PgSPL3, PgSPL6, PgSPL11 and PgSPL14 in leaves were significantly higher than those in buds and stems $(p<0.05)$. The expression levels of PgSPL5, PgSPL12 and PgSPL13 in flower buds were significantly higher than that in leaves and stems $(p<0.05)$. The full-length of coding sequence of PgSPL5 and PgSPL13 were obtained by homologous cloning technology. The full length of PgSPL5 is 1020 bp, and PgSPL13 is 489 bp, which encodes 339 and 162 amino acids, respectively. Further investigation revealed that PgSPL5 and PgSPL13 proteins were located in the nucleus. Exogenous plant growth regulator induction experiments showed that PgSPL5 was up-regulated in leaves and stems. PgSPL13 was up-regulated in leaves and down-regulated in stems. When sprayed with 6-BA, IBA and PP333 respectively, PgSPL5 and PgSPL13 were up-regulated most significantly at P2 (bud vertical diameter was $5.1 \sim 12.0$ $\mathrm{mm})$ stage of bisexual and functional male flowers.

Conclusions: Our findings suggested that PgSPL2, PgSPL3, PgSPL6, PgSPL11 and PgSPL14 played roles in leaves development of pomegranate. PgSPL5, PgSPL12 and PgSPL13 played roles in pomegranate flower development. PgSPL5 and PgSPL13 were involved in the response process of different plant hormone signal transduction in pomegranate development. This study provided a robust basis for further functional analyses of SPL genes in pomegranate.
\end{abstract}

Keywords: Pomegranate, SPL gene family, Gene cloning, Subcellular localization, Gene expression

\footnotetext{
* Correspondence: zhyuan88@hotmail.com

${ }^{1}$ Co-Innovation Center for Sustainable Forestry in Southern China, Nanjing Forestry University, Nanjing 210037, China

${ }^{2}$ College of Forestry, Nanjing Forestry University, Nanjing 210037, China
}

\section{$\triangle B M C$}

(c) The Author(s). 2021 Open Access This article is licensed under a Creative Commons Attribution 4.0 International License, which permits use, sharing, adaptation, distribution and reproduction in any medium or format, as long as you give appropriate credit to the original author(s) and the source, provide a link to the Creative Commons licence, and indicate if changes were made. The images or other third party material in this article are included in the article's Creative Commons. licence, unless indicated otherwise in a credit line to the material. If material is not included in the article's Creative Commons licence and your intended use is not permitted by statutory regulation or exceeds the permitted use, you will need to obtain permission directly from the copyright holder. To view a copy of this licence, visit http://creativecommons.org/licenses/by/4.0/ The Creative Commons Public Domain Dedication waiver (http://creativecommons.org/publicdomain/zero/1.0/) applies to the data made available in this article, unless otherwise stated in a credit line to the data. 


\section{Background}

Transcription factors can specifically bind with cis-acting elements of the promoter, which activate or suppress the expression of downstream target genes. The transcription factors involved in floral organ development mainly include MADS-box [1], bHLH [2], YABBY [3] and SPL [4]. The plant-specific SPL (SQUAMOSA promoter binding protein-like) transcription factor is involved in the formation and development of flowers, leaf morphogenesis, and other biological processes [5, 6]. It is known that SPL family members have a highly conserved SBP domain consisted of 79 amino acids, and have two typical zinc finger structures including $\mathrm{C} 3 \mathrm{H}(\mathrm{C}-\mathrm{C}-\mathrm{CH})$ and $\mathrm{C} 2 \mathrm{HC}(\mathrm{C}-\mathrm{C}-\mathrm{H}-\mathrm{C})$ [7]. The C-terminal of the SPL proteins combine with the second zinc finger promoting its combination with the DNA to perform function [8].

SPL family genes were originally isolated from $A$. majus [9]. They regulate early flowering of plants by binding with the SQUAMOSA promoter of MADS-box family genes [9]. In Arabidopsis, 17 SPL genes have been isolated and named as SPL1 SPL16. AtSPL13 are a pair of paralogous genes, which are named as SPL13A and $S P L 13 B$ [10]. In recent years, many researchers have successively identified SPL family genes in other species, such as 28 SPL genes in poplar (Populus trichocarpa) [11], 15 in tomato (Solanum lycopersicum) [12], 19 in rice (O. sativa) [13], 27 in apple (M. domestica Borkh.) [14] and 18 SPL genes in grape (Vitis vinifera L.) [15].

MicroRNAs (miRNAs) are a class of endogenous noncoding RNAs, about $20-24$ nt in length, that are derived from primary miRNA transcripts (pri-miRNAs) containing a stem-loop secondary structure $[16,17]$. They are known to suppress the expression of target genes at the post-transcriptional level via mRNA cleavage or translational inhibition $[18,19]$. In plants, miRNAs act crucial roles in plant organ development [20], stress tolerance [21], phytohormone signaling [22], growth phase change [23], and disease resistance [21]. miR156 is a highly conserved class of miRNAs, which involves in regulating the transformation of plants from vegetative stage to reproductive stage [24-26]. Among 17 SPL genes in Arabidopsis, 11 members have the miR156 binding sites [2730]. According to the size of the SBP domain, the $S P L$ genes regulated by miR156 can be divided into two categories. One includes SPL3, SPL4, and SPL5, which encode smaller proteins and promote flower formation; the other of SPL9 and SPL15 encode larger proteins, which mainly promote leaf formation and flowering $[31,32]$.

Previous studies found that SPL2 regulates floral organ development and plant fertility by activating $A S Y M M E T$ RIC LEAVES 2 (AS2) [33]. SPL3/4/5 can activate the expression of LEAFY (LFY), FRUITFULL (FUL) and APETALA1 (AP1) in floral meristems [34, 35]. As a gene not regulated by miR156, SPL8 plays a redundant role with other miR156-targeted SPL genes, which can regulate the development of microspore sac, megasporogenesis, hairy body formation on sepals, and stamen filament elongation, so as to control the male fertility of plants [36]. SPL9 and SPL10 control flowering time by directly activating miR172 [28]. SPL15 is a positive regulator for flowering stage, and it cooperates with SUPPRESSOR OF CONSTANS 1 (SOC1) to induce Arabidopsis flower development [37]. SPL2, SPL8, SPL9 and SPL15 have the effect of maintaining male fertility in Arabidopsis [38, 39].

Pomegranate is an excellent tree species with nutritional, medicinal, ornamental and ecological values [40]. Studies have confirmed that SPL factors play an important role in floral transition and flower development [32, 34, $35,37,41]$. Whether it plays a role in pomegranate development has not been reported. Based on the whole genome data of 'Taishanhong' pomegranate, the members of SPL family were identified by bioinformatics methods. Their physical and chemical characteristics were analyzed. The evolution relationship, gene structure, conserved motifs, difference of cis-acting elements and expression of gene tissue characteristics of each member were explored. The target sites of miR156 were predicted. The temporal and spatial expression characteristics of PgSPL5 and PgSPL13 genes under the treatment of cloning, subcellular localization and growth regulator were analyzed to provide reference for revealing their biological functions in pomegranate flower induction.

\section{Results}

\section{Identification and characterization of SPL transcription} factors

Fifteen SPL gene family members were identified from the genome of 'Taishanhong' pomegranate. For the convenience of subsequent analysis, they were named as PgSPL1 PgSPL15 (Table 1). The protein sequence and physicochemical properties of PgSPL members were analyzed. The results showed that the length of PgSPL genes changed greatly. The shortest protein encoded 162 amino acids (PgSPL13), the longest is 1049 amino acids (PgSPL9), and the molecular weight of the protein ranged from $18,563.02 \mathrm{kDa}$ to $115,942.26 \mathrm{kDa}$. The theoretical isoelectric point ranged from 5.38 to 9.39 , with PgSPL13 as low as 5.38 and PgSPL14 as high as 9.39. It contains 10 acidic proteins and 5 basic proteins. These results provided a theoretical basis for further purification, activity and function research of PgSPL proteins. The results of subcellular location showed that members were expressed in the nucleus, except PgSPL4, PgSPL6 and PgSPL12 in the nucleus and cytoplasm.

\section{Phylogenetic analysis}

Multiple sequence alignment results (Fig. 1) showed that each SPL transcription factor family member contained 
Table 1 Member characteristics of SPL gene family in pomegranate

\begin{tabular}{llllllll}
\hline Gene Name & Gene ID & Location & Length & pl & Mw(kDa) & Group & Subcellular Localization \\
\hline PgSPL1 & Pg000744.1 & scaffold1:4774751:4776860 & 500 & 6.37 & $54,138.16$ & G3 & Nuclear \\
PgSPL2 & Pg003831.1 & scaffold12:3285330:3286080 & 196 & 9.16 & $21,938.35$ & G5 & Nuclear \\
PgSPL3 & Pg005590.1 & scaffold13:3198475:3200620 & 527 & 8.87 & $57,576.09$ & G3 & Nuclear \\
PgSPL4 & Pg008456.1 & scaffold16:1476693:1481665 & 778 & 5.53 & $86,532.31$ & G1 & Cytoplasm/Nucleur \\
PgSPL5 & Pg014414.1 & scaffold25:1722378:1724406 & 339 & 8.89 & $36,911.79$ & G4 & Nuclear \\
PgSPL6 & Pg016042.1 & scaffold3:3668488:3670385 & 389 & 8.87 & $41,945.73$ & G2 & Cytoplasm/Nucleur \\
PgSPL7 & Pg016049.1 & scaffold3:3581401:3584942 & 493 & 8.4 & $54,118.39$ & G3 & Nuclear \\
PgSPL8 & Pg017676.1 & scaffold33:999708:1004761 & 999 & 6.76 & $111,303.95$ & G6 & Nuclear \\
PgSPL9 & Pg019080.1 & scaffold39:1554086:1558442 & 1049 & 8.06 & $115,942.26$ & $G 6$ & Nuclear \\
PgSPL10 & Pg020599.1 & scaffold42:1057229:1061842 & 986 & 5.92 & $109,247.27$ & G6 & Nuclear \\
PgSPL11 & Pg027304.1 & scaffold72:239136:241212 & 391 & 8.61 & $42,020.97$ & G2 & Nuclear \\
PgSPL12 & Pg028546.1 & scaffold80:561916:564888 & 402 & 9.4 & $42,499.1$ & G3 & Cytoplasm/Nucleur \\
PgSPL13 & Pg029123.1 & scaffold84:570880:572840 & 162 & 5.38 & $18,563.02$ & G5 & Nuclear \\
PgSPL14 & Pg029352.1 & scaffold87:784733:785930 & 171 & 9.39 & $19,317.66$ & G5 & Nuclear \\
PgSPL15 & Pg030861.1 & scaffold99:46274:51496 & 334 & 8.9 & $36,242.96$ & G3 & Nuclear
\end{tabular}

Mw molecular weight, $p$ isoelectric points

a highly conserved SBP domain composed of 79 amino acid residues. They all contain two zinc finger structures Cys-Cys-Cys-His (C3H), Cys-Cys-His-Cys (C2HC) and a nuclear localization signal (NLS). The $\mathrm{N}$-terminal zinc finger structure of PgSPL4 is Cys4 (C4), which is different from other members.

The phylogenetic tree results showed that 15 SPL genes of pomegranate were clustered into six subfamilies (G1 G6) (Fig. 2) [42, 43]. Among these six subgroups, G1 subgroup has a distant genetic relationship with other subgroups, forming a relatively independent branch. Further analysis found that the Nterminal zinc finger structure in the SBP domain of the five members of the G1 subgroup AtSPL7, PgSPL4, VvSPL6, MdSPL17, and MdSPL25 is C4, while that of all members in the other subgroups is

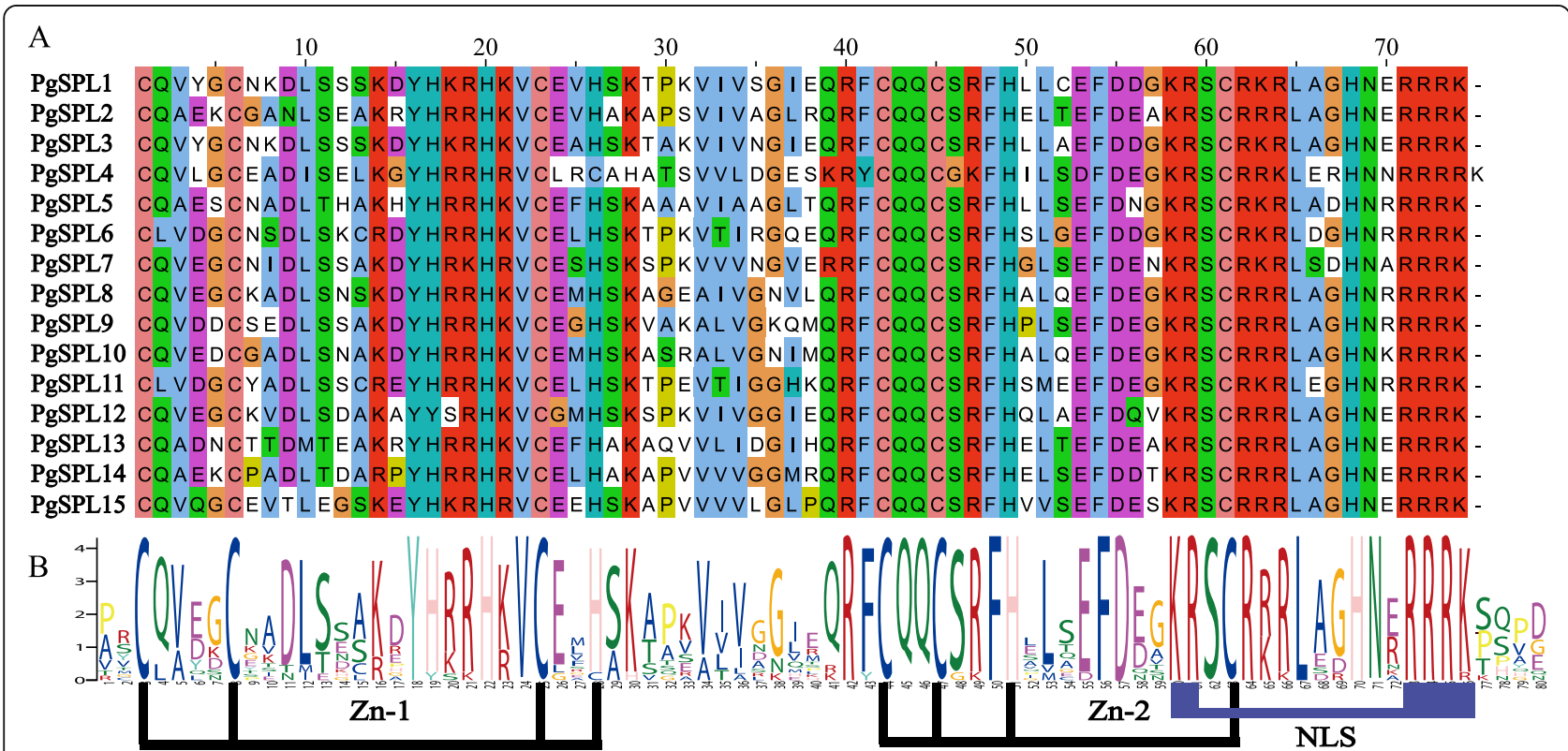

Fig. 1 Multiple alignment of the SBP domains of the pomegranate SPL proteins. a Multiple alignment of the SBP domains of the PgSPL proteins was obtained with DNAMAN software. The two conserved zinc finger structures (Zn-1, Zn-2) and NLS are shown. b Sequence logo of the SBP domain of PgSPL proteins. The height of the letters within each stack represents the relative frequency of the corresponding amino acids 


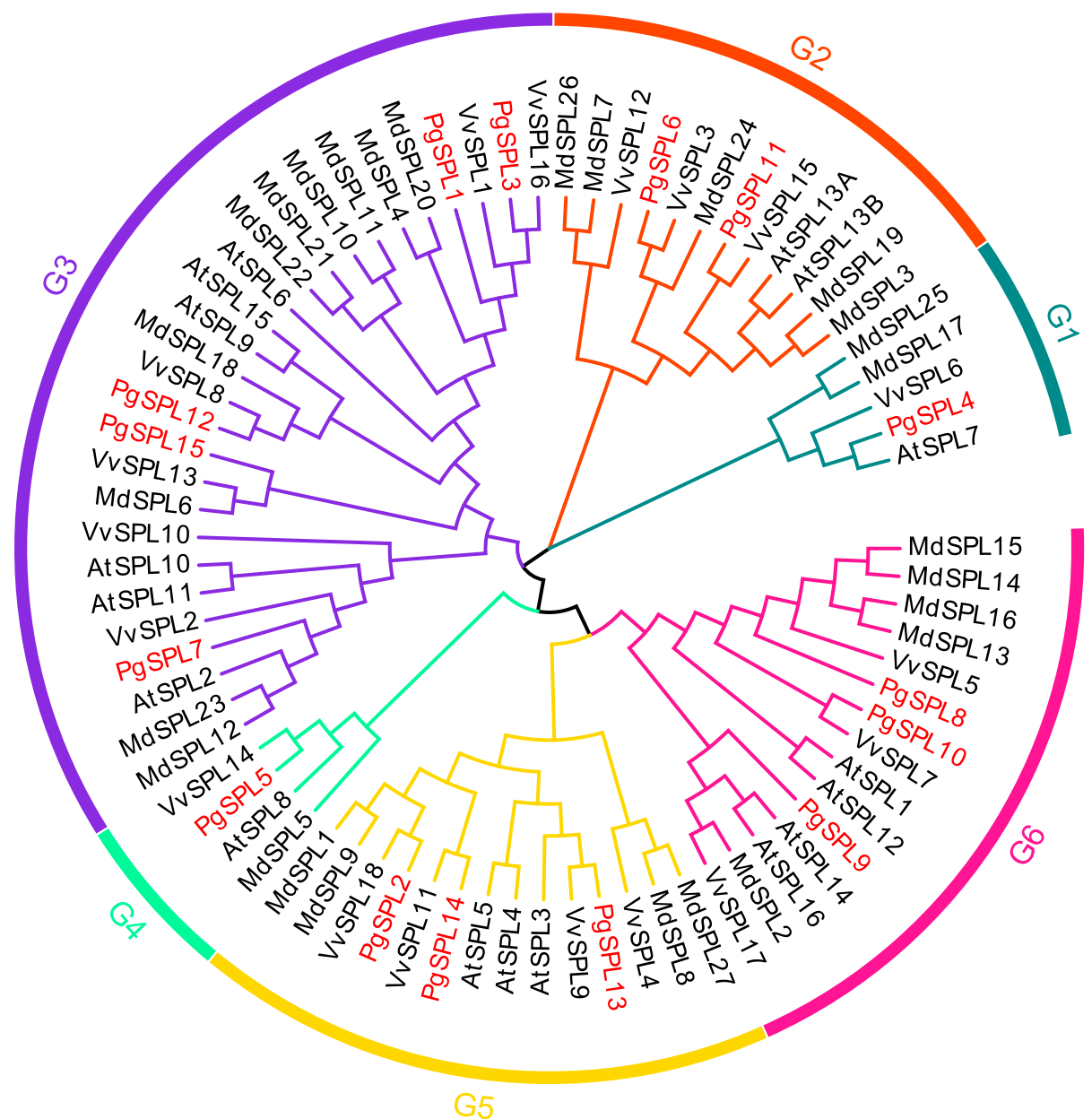

Fig. 2 Phylogenetic tree of four species: Punica granatum, Arabidopsis, M. domestica and Vitis vinifera

$\mathrm{C} 3 \mathrm{H}$. The members of SPL transcription factor family in G3 subgroup were the most widely distributed. Among them, 5 of 15 pomegranate SPL genes were distributed in G3 subgroup. G4 subgroup each has a pomegranate, Arabidopsis, apple and grape SPL gene. There were two PgSPL genes in G2 subgroup, and three PgSPL genes in G5 and G6.

\section{Gene structure analysis and motif identification of PgSPLs}

The results of PgSPLs gene structure showed that the number of intron was $1 \sim 9$ (Fig. 3c). The members of G1 and G6 contained nine introns. In the G2, G3 and G4 subgroups, the members had 2 introns except PgSPL7 possessed 3 introns. PgSPLs in G5 subgroup contained only one intron.

Ten conserved motifs of PgSPLs were identified (Fig. 3b). All PgSPL proteins contained Motif1, the SBP domain, which is composed of about 79 amino acids. PgSPLs members in the same subfamily showed similar gene structure and protein conservation motif. In G2 G5 subgroups, all members only contained the SBP domain, except PgSPL11. However, members in G1 and G6 subgroups have some no or atypical domains in other subgroups, the differential distribution of these conserved motifs might be the reasons for gene functional difference.

\section{Cis-acting elements located in SPL gene promoters}

The cis-acting elements contained a wide variety, ranging from 23 in PgSPL14 to 42 in PgSPL7 (Fig. 4). PgSPLs respond to different plant hormones and abiotic stress signals, including AAGAA-motif and ABRE, the cis-acting elements involved in abscisic acid response, gibberellin response element P-box, methyl jasmonate response element CGTCA-motif and TGACG-motif, the necessary cis-regulatory element ARE for anaerobic induction, the cis-acting regulatory element CAT-box which is related to meristem expression, the cis-acting regulatory element G-Box which is involved in photoreactivity, the cold stress response element LTR, the droughtinduced response element MBS, and the common 


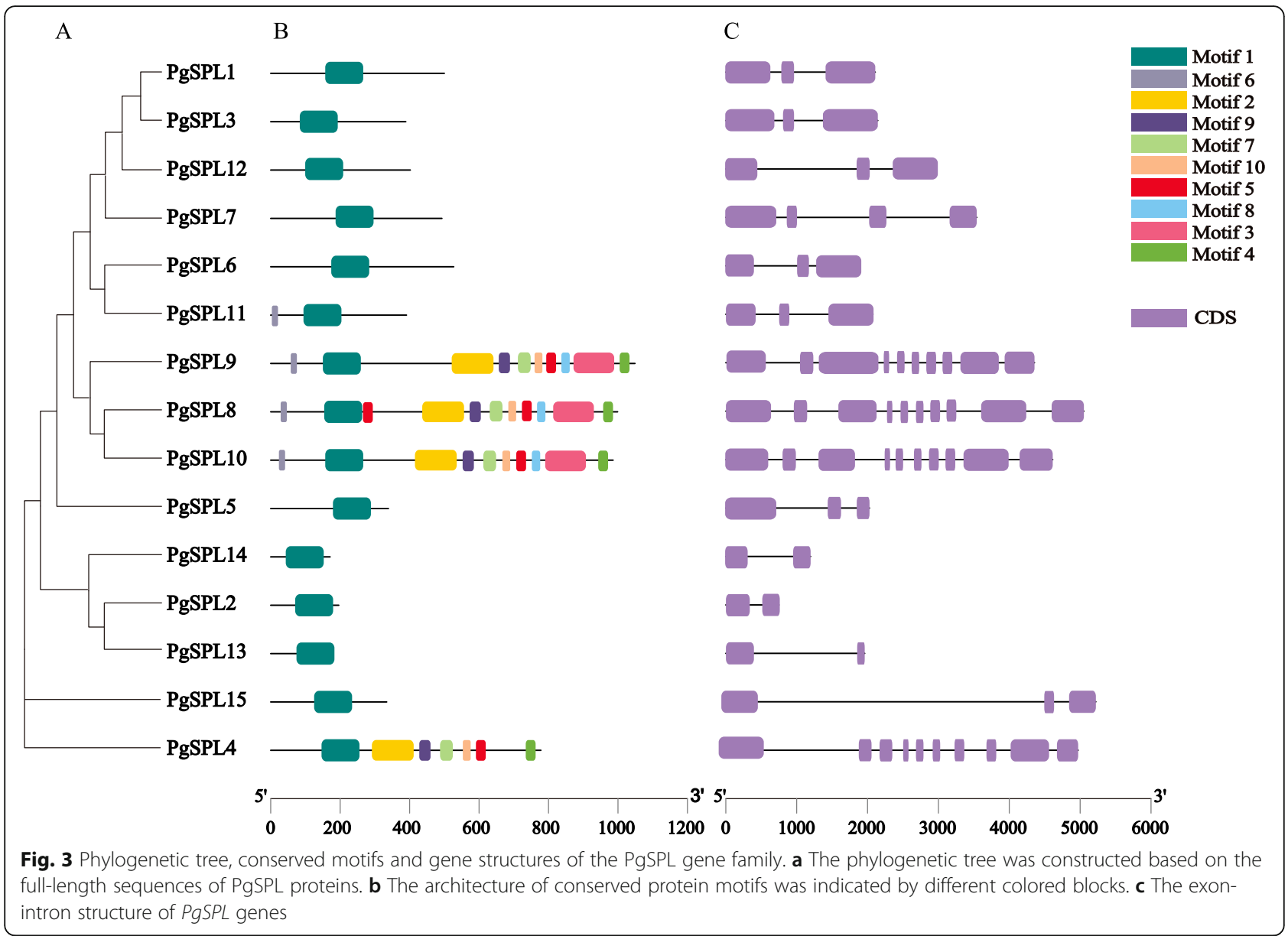

cis-acting element CAAT-box in the promoter and enhancer regions. Among 15 PgSPLs, 11 genes contained AAGAA-motif and ABRE elements, 7 genes contained P-box elements, and 8 contained CGTCAmotif and TGACG-motif elements. Ten members contained ARE elements, 9 members contained CAT-box elements, 5 genes contained G-Box elements, 9 genes contained stress-related elements LTR and MBS, and all PgSPL genes contained enhancer elements CAAT-box.

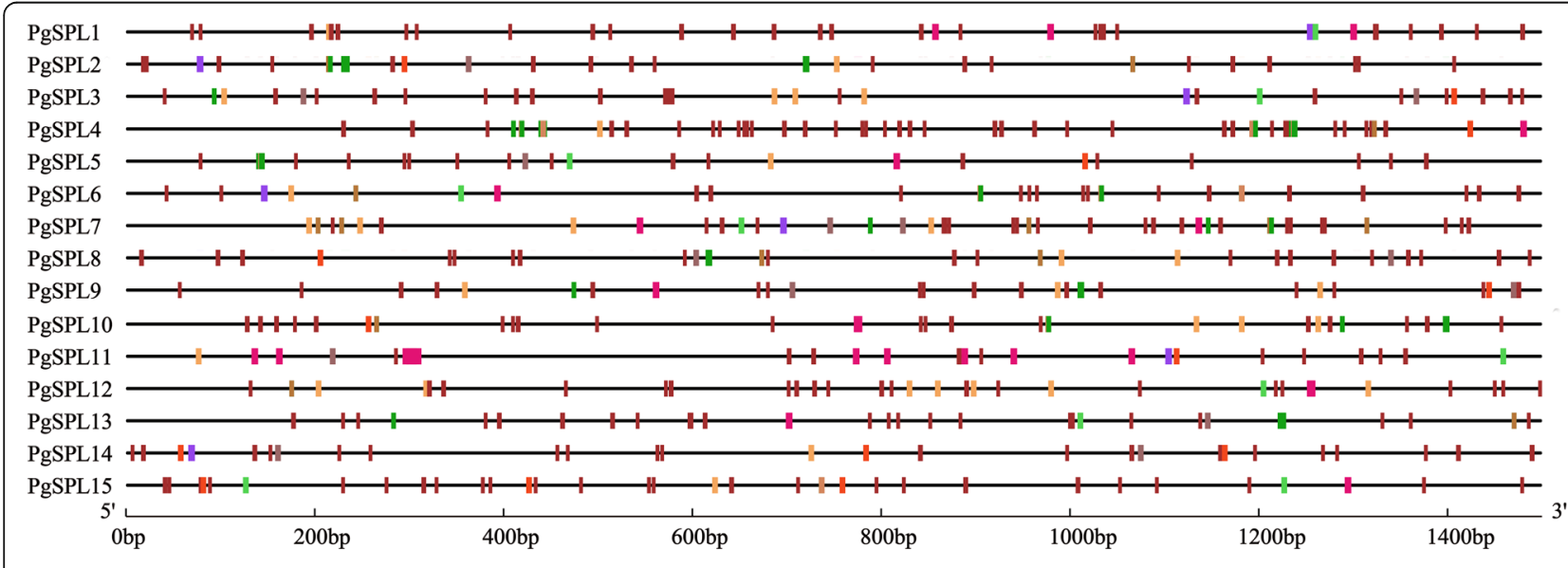

AAGAA-motif $\square$ ABRE $\square$ ARE $\square$ CAAT-box $\square$ CAT-box $\square$ CGTCA-motif $\square$ G-Box $\square$ LTR $\square$ MYB $\square$ P-box $\square$ TGACG-motif

Fig. 4 Cis-elements analysis of pomegranate SPL genes 
Prediction of PgSPL genes targeted by miR156

Many reserches have shown that most SPL genes are regulated by miR156, and miR156 target sites are located in the coding region or 3'UTR region. In order to determine post-transcriptional regulatory mechanism in pomegranate, miR156 target sites were searched at the coding regions and 3'UTR regions of PgSPLs. The results showed that PgSPL1, PgSPL2, PgSPL3, PgSPL6, PgSPL7, PgSPL11, PgSPL12, PgSPL13, PgSPL14 and PgSPL15 were potential targets for miR156, and these genes were clustered in G2, G3 and G5 subgroups. The PgSPLs with potential miR156 targets in the coding region belong to G2 and G3, while PgSPL2, PgSPL13 and PgSPL14 with targets sites in 3'UTR belong to the G5 subgroup. PgSPL gene sequences were compared with the mature PgmiR156 sequence, and the results showed that more than half of PgSPL contained sequences complementary to PgmiR156 sequence with no more than two mismatched bases (Fig. 5). This result is similar to other species $[43,44]$.

\section{Expression patterns by transcriptome data}

As shown in Fig. 6, comparative analysis of the transcriptome was in different tissues. The results showed that the expression of PgSPL1 and PgSPL3 were high in pericarp, and were highly expressed in flowers, more than those in seed coat. PgSPL2 expression was high in flowers, moderate in inner and outer seed coat, but not in 'Wonderful' pericarp. There was no significant difference in PgSPL4 expression levels in all tissues. PgSPL5 showed high expression level in the stages of bisexual flowers I $(3.0 \sim 5.0 \mathrm{~mm})$, functional male flowers I $(3.0 \sim$ $5.0 \mathrm{~mm})$ and II $(5.1 \sim 13.0 \mathrm{~mm})$, and lower in inner seed coat. PgSPL6 is expressed at a low level in the outer seed coat. PgSPL7 showed higher expression in the outer seed coat and bisexual flowers II (5.1 $13.0 \mathrm{~mm})$. PgSPL8 and
PgSPL9 exhibited similar patterns of expression. PgSPL10 was highly expressed in 'Wonderful' pericarp. PgSPL11 and PgSPL12 were not expressed in 'Wonderful' pericarp. PgSPL13 was expressed at the highest level in leaves, maintained at a high level during the stage I and II of bisexual flowers and functional male flowers, but low expression in outer seed coat. PgSPL14 had the highest expression levels of functional male flowers I and III. The highest level of PgSPL15 was found in leaves, while the expression was low or not expressed in other tissues.

We also validated miR156a-5p and PgSPL13 that have differentially expression patterns during the different stages of pomegranate flowers development (Fig. 7). It was found that during the development of bisexual flowers (Fig. 7a), the expression of miR156a-5p first increased and then decreased, while the expression of PgSPL13 first decreased and then increased. And the expression was significantly different in each period $(p<$ 0.05). During the development of functional male flowers (Fig. 7b), the expression of miR156a-5p was significantly decreased, while the expression of PgSPL13 was significantly increased $(p<0.05)$.

\section{qRT-PCR analysis of PgSPLs}

From Fig. 6, eight differentially expressed PgSPLs genes were screened out, the expression patterns of PgSPL genes in different tissues of pomegranate was analyzed by qRT-PCR. The results are shown in Fig. 8, the expression levels of PgSPL2 were the highest at P7 and P8 stages of two types of flowers, and the expression levels at P5 stage of the bisexual flowers were significantly lower than those at other stages $(p<0.05)$. We found that the expression levels of PgSPL3 in P4 P6 stages of bisexual flowers were significantly lower than those in other stages. It was significantly increased in P6 P8 stages $(p<0.05)$,

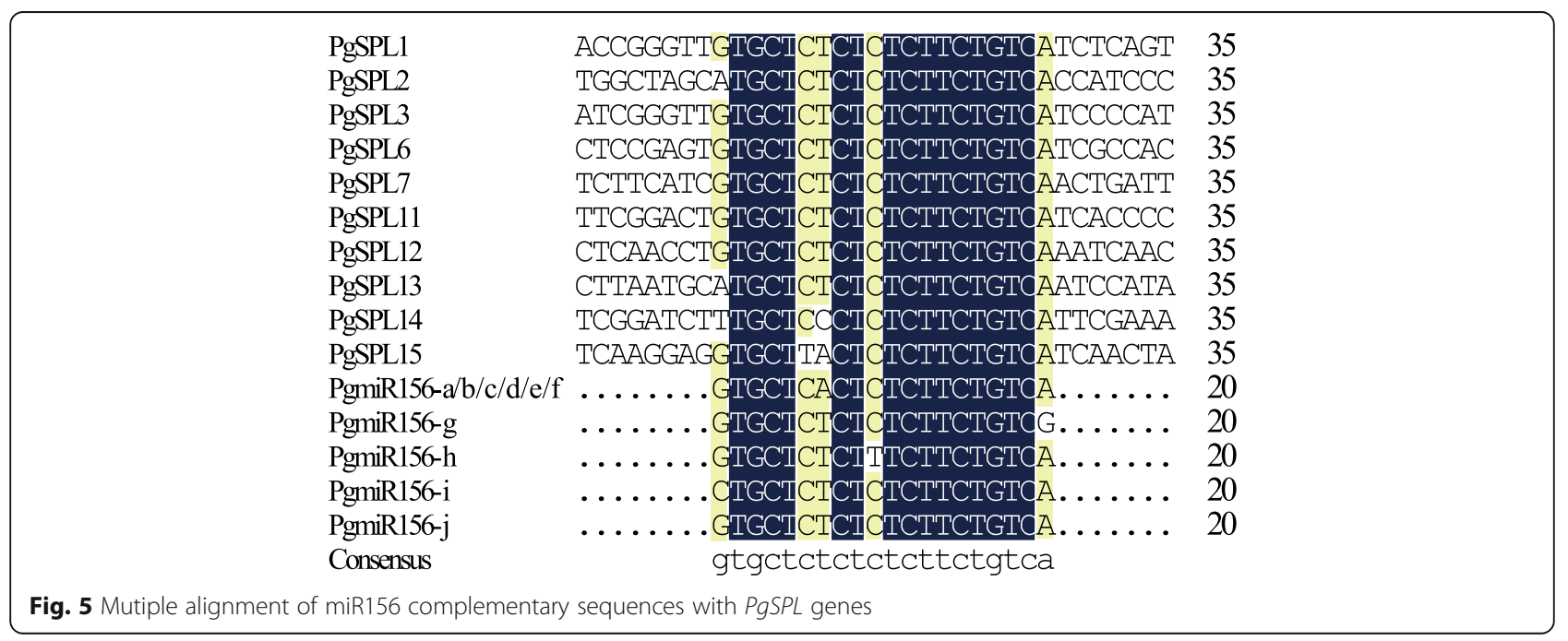




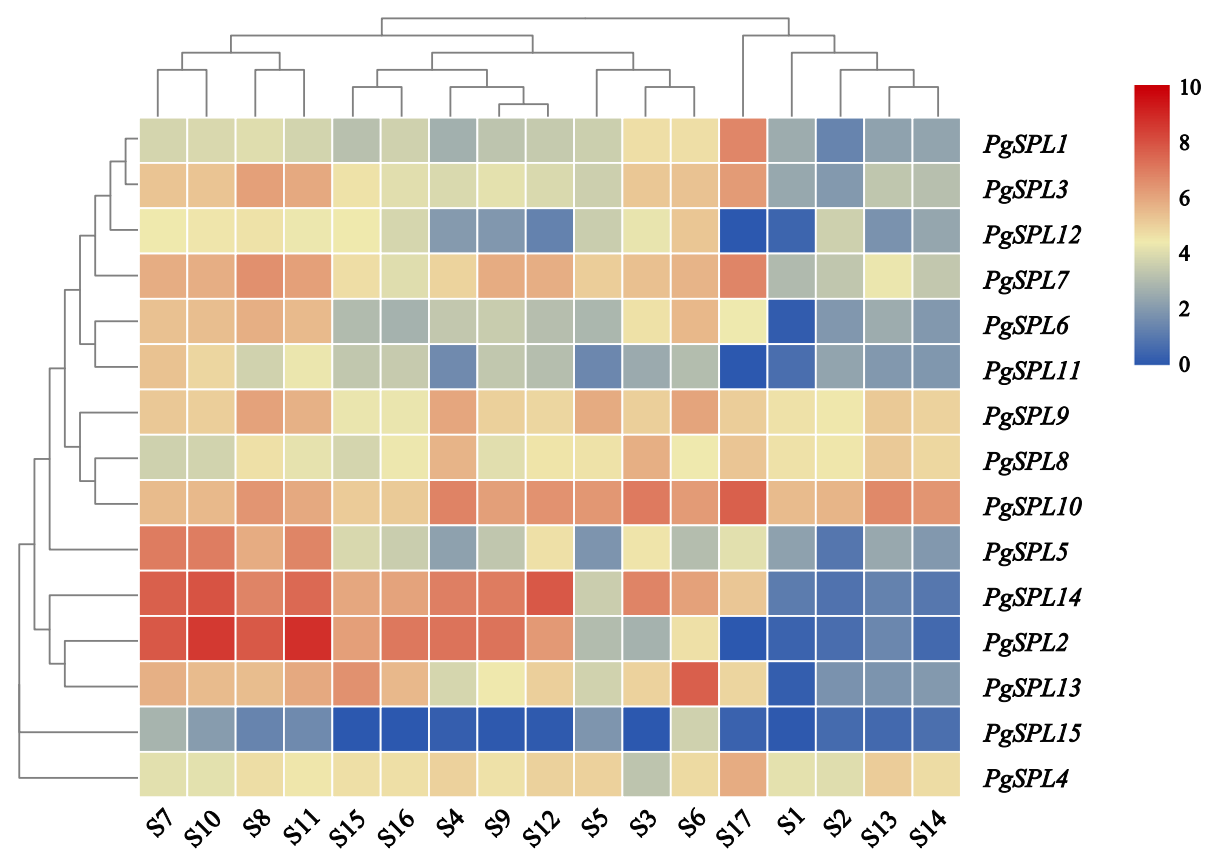

Fig. 6 The heatmap of expression of SPL genes in different tissues of pomegranate. S1: Outer seed coat; S2: Inner seed coat; S3: Pericarp; S4; Flower; S5: Root; S6: Flesh leaf; S7: Bisexual flowers (3.0 $5.0 \mathrm{~mm})$; S8: Bisexual flowers (5.1 13.0 mm); S9: Bisexual flowers (13.1 25.0 mm); S10: Functional male flowers $(3.0 \sim 5.0 \mathrm{~mm})$; S11: Functional male flowers $(5.1 \sim 13.0 \mathrm{~mm})$; S12: Functional male flowers $(13.1 \sim 25.0 \mathrm{~mm})$; S13: Inner seed coat of 'Tunisia'; S14: Inner seed coat of 'Baiyushizi'; S15: Mix of leaves, flowers, fruit and roots of 'Black127'; S16: Mix of leaves, flowers, fruit and roots of 'nana'; S17: Peels of 'Wonderful' (cultivars S1 S6 are 'Dabenzi', cultivars S7 S13 are 'Tunisia')

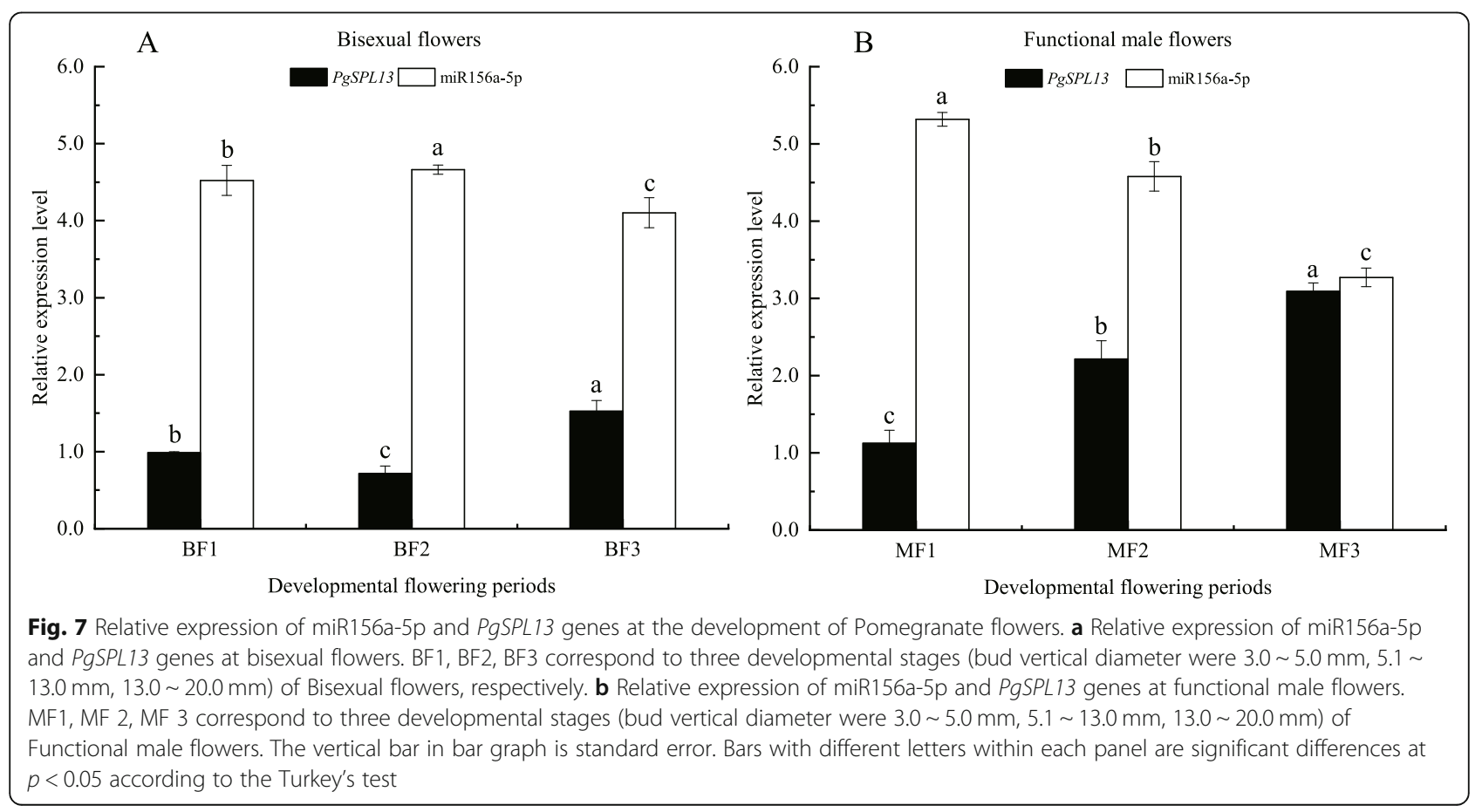




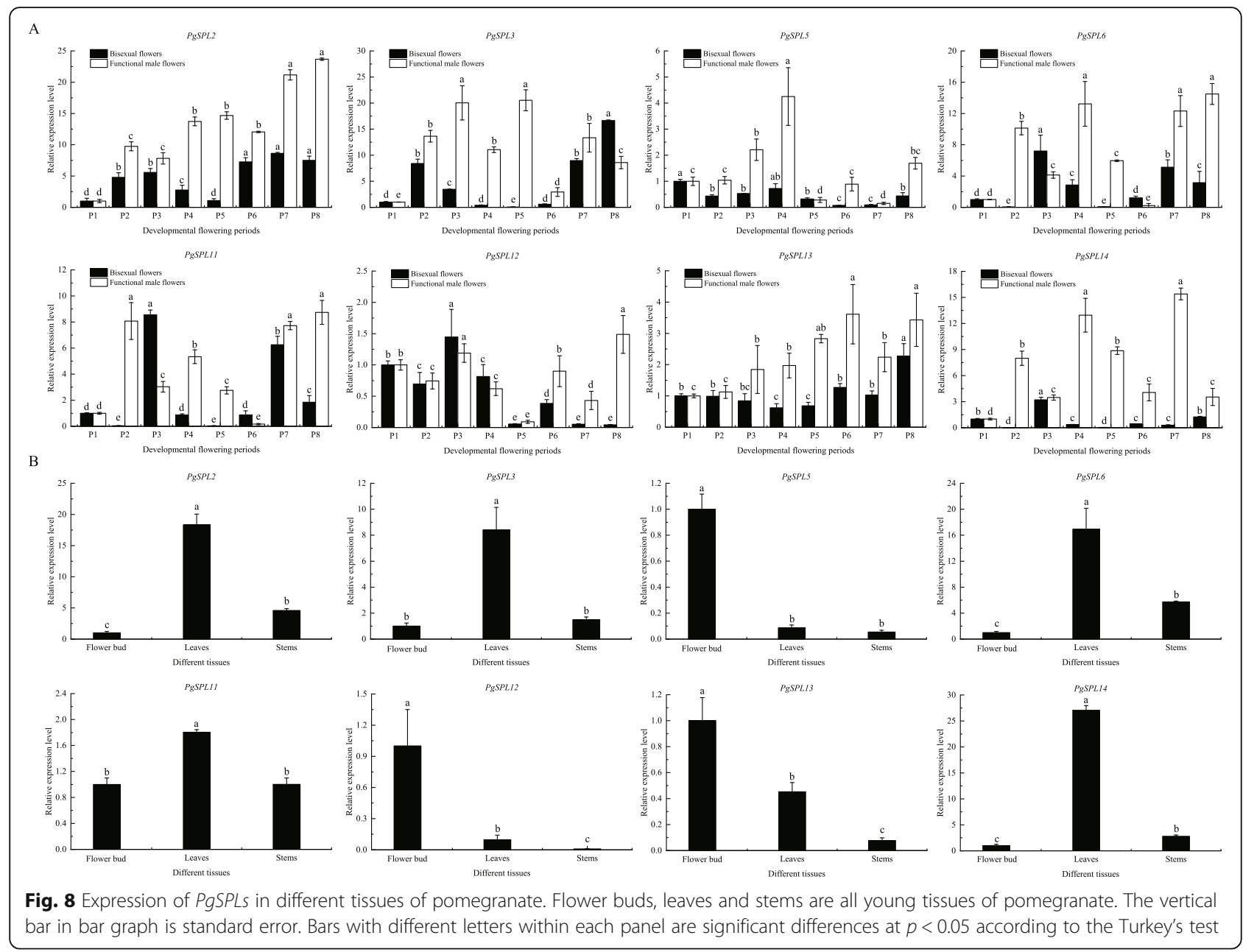

and it was higher in P2 P5 stages of functional male flowers. PgSPL5 had higher expression in functional male flowers than in bisexual flowers, and its expression level showed a trend of up-down-up during the development of bisexual flowers. At P2 $\sim$ P4 stages of functional male flowers, the expression levels of PgSPL5 were significantly increased $(p<0.05)$, and reached the highest level at the P4 stage, then slightly downregulated. The expression levels of PgSPL6 in the P2 and P5 stages of bisexual flowers were significantly lower than those in other stages $(p<0.05)$. The expression levels of PgSPL6 in the P8 stage of functional male flowers were the highest, followed by those in the P4 and P7 stages, and then the P6 stage. A significantly greater amount of PgSPL11 was expressed in the P6 stage of the bisexual flowers compared with that in the other stages $(p<0.05)$, and its expression was lowest at P5 stage. In the P2, P7 and P8 stages of functional male flowers, the expression levels of PgSPL11 were significantly higher than that in other stages $(p<0.05)$. The expression levels of PgSPL12 were higher in P2 P4 stages of two kinds of flowers, and showed a consistent trend. The expression levels in P5, P7 and P8 stages of amphoteric flowers were significantly lower than that in other stages $(p<0.05)$, and the expression level was the highest in P8 stage of functional male flowers. The expression levels of PgSPL13 dropped first and then went up during the development of bisexual flowers, which showed the opposite trend in functional male flowers. PgSPL13 showed higher expression in functional male flowers compared with bisexual flowers, reaching a maximum value in P8 stage of the bisexual flower and P6 stage of the functional male flower. The expression level of PgSPL14 was higher in P3 stage and lower in other stages. In functional male flowers, the expression levels of PgSPL14 were the highest in P4 and P7 stages, followed by P2 and P5 stages.

It can also be seen from Fig. 8 that the expression levels of PgSPL2, PgSPL13 and PgSPL14 in functional male flowers at P1 $\sim$ P8 stages were higher than those in bisexual flowers. The expression levels of PgSPL2, PgSPL3, PgSPL6, PgSPL11 and PgSPL14 in young leaves were significantly higher than those in buds and stems $(p<0.05)$. The expression levels of PgSPL5, PgSPL12 and PgSPL13 in flower buds were significantly higher than that in leaves and stems $(p<0.05)$. 
Cloning and sequence analysis of PgSPL5 and PgSPL13

PgSPL5 and AtSPL8 were clustered into the same group in the phylogenetic trees, and the reconstructed phylogenetic tree also showed that PgSPL13 was on the same branch as AtSPL3. Studies have shown that AtSPL8 played an important role during anther development [36], and AtSPL3 had a very important function in the development of Arabidopsis flowers $[34,35]$. Therefore, we speculated that PgSPL5 and PgSPL13 also play roles in pomegranate flower development. Figure 8 showed that the expression levels of PgSPL5 and PgSPL13 in flower buds were significantly higher than those in leaves and stems $(p<0.05)$, which further indicated that these two genes have important functions in pomegranate flower development. Accordingly, we chose these two genes for cloning. Using 'Taishanhong' pomegranate cDNA as templates, PgSPL5 primers and PgSPL13 primers were used for PCR amplification respectively. The electrophoresis bands were consistent with the expected sizes of the target fragments (Fig. 9). The coding region sequences of PgSPL5 and PgSPL13 were obtained by sequencing, which were $1020 \mathrm{bp}$ and $489 \mathrm{bp}$, encoding 339 and 162 amino acids, respectively. The molecular weight of the proteins was predicted as $36,911.79 \mathrm{kDa}$ and $18,563.02 \mathrm{kDa}$, respectively. Both genes contained a complete SBP conserved domain (Fig. 10).

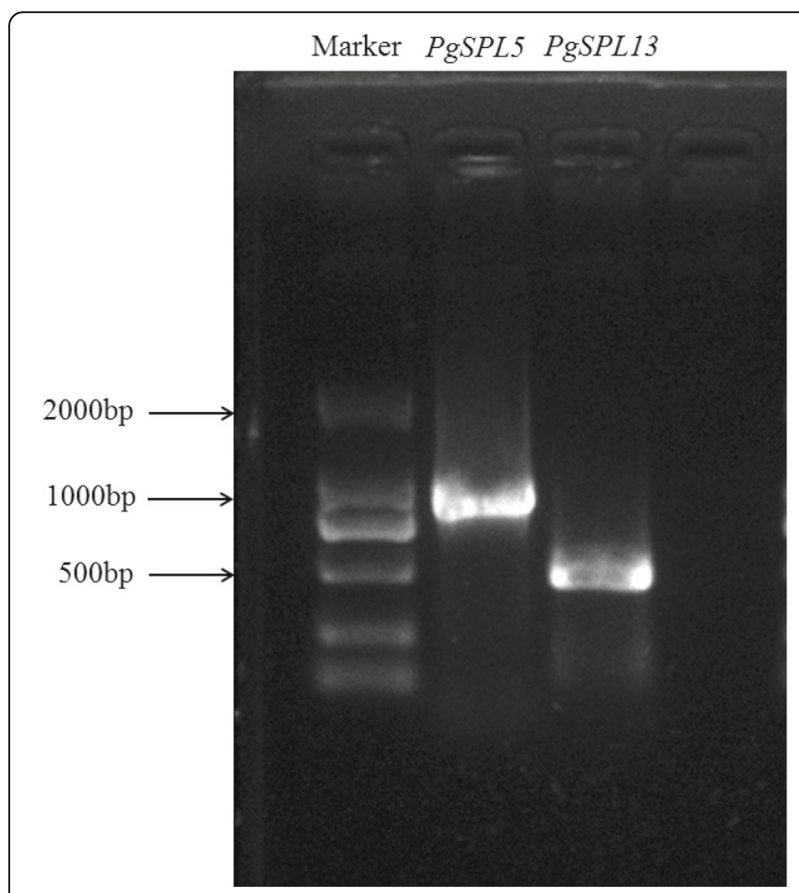

Fig. 9 PCR amplification of PgSPL5 and PgSPL13 genes. Excess gel is cropped in the image
Subcellular localization of PgSPL5 and PgSPL13

The subcellular localization of PgSPL5 and PgSPL13 was predicted by using the online tool Cell-PLoc [45], and it was found that they were both located in the nucleus. In order to verify the above predicted results, we transiently expressed 35S::GFP-PgSPL5 and 35S::GFP-PgSPL13 fusion proteins in tobacco leaves, and found that these two genes were indeed located in the nucleus (Fig. 11). It was speculated that PgSPL5 and PgSPL13 might be involved in the regulation of gene expression.

\section{Effects of exogenous hormones on expression of PgSPL5 and PgSPL13}

It was found that proper spraying of plant growth regulators could promote flower bud differentiation, induce female flower formation, increase plant stress resistance and increase yield. To investigate whether the expression patterns of PgSPL5 and PgSPL13 genes were affected by different growth regulators, the detailed expression patterns in response to different treatments were analysed by qRT-PCR and showed a great variation among different treatment groups.

As shown in Fig. 12a and b, the expression level of PgSPL5 was significantly up-regulated at the P2 stage of the two types of flowers under 6-BA treatment, and the expression was up-regulated but not significant at the P7 stage of bisexual flowers. In other developmental stages of bisexual flowers, the expression of PgSPL5 was downregulated after exogenous 6-BA treatment, and PgSPL5 expression was significantly down-regulated at the P3, P4 and P8 stages of functional male flowers. Significantly increased expression of PgSPL5 was observed in leaves and stems under 6-BA treatment (Fig. 12g), and this change was more pronounced in stems. PgSPL13 was up-regulated at P2 stage of bisexual flowers, and downregulated at all other stages after 6-BA treatment. The expression of PgSPL13 in leaves was significantly upregulated under 6-BA treatment, while in stems it was down-regulated (Fig. 12h).

After IBA treatment (Fig. 12c, d), we found that the expression of PgSPL5 was down-regulated at the P3 and P8 stages of bisexual flowers, and up-regulated at other developmental stages of bisexual flowers, while the upregulation was extremely significant at the P2 stage. At P2 and P5 stages of functional male flowers, the expression levels of PgSPL5 were significantly up-regulated, but down-regulated at other stages under IBA treatment. In leaves and stems, the expression of PgSPL5 under IBA treatment showed the same change as 6-BA treatment, but this change was not as obvious as 6-BA treatment. PgSPL13 was significantly up-regulated in P2 and P5 stages of bisexual flowers and P2 and P4 stages of functional male flowers, and down-regulated at other stages of flower development after IBA treatment. Similarly, 


\begin{tabular}{|c|c|c|c|c|c|c|c|c|c|c|c|c|c|c|c|c|c|c|c|c|c|}
\hline A & \multirow[t]{2}{*}{1} & atg & $\operatorname{ttg}$ & gac & \multicolumn{2}{|c|}{ tat gaa } & & gct & get & $g c g$ & aac & $\operatorname{ccc}$ & tct & \multicolumn{2}{|r|}{ gtg } & atg & ctc & $\operatorname{tcg}$ & gcc & gac & gat \\
\hline & & M & I & $\mathrm{D}$ & $\mathrm{Y}$ & $\mathrm{E}$ & $\mathrm{W}$ & $\mathbb{A}$ & A & $\mathrm{A}$ & $\mathrm{N}$ & P & $s$ & $s$ & $\mathrm{~V}$ & M & L & $s$ & A & D & $\mathrm{D}$ \\
\hline & 61 & $\operatorname{ccc}$ & gct & tgc & $\operatorname{ccc}$ & gat & $\operatorname{ccc}$ & $\operatorname{tcc}$ & cat & $g g t$ & cac & $\operatorname{ccc}$ & tet & gtc & gac & cac & tat & gcc & gca & $g c c$ & act \\
\hline & & P & A & c & P & D & $\mathrm{P}$ & $s$ & $\mathrm{H}$ & G & $\mathrm{H}$ & $\mathrm{P}$ & F & $\mathrm{v}$ & D & $\mathrm{H}$ & $Y$ & $\mathrm{~A}$ & $\mathrm{~A}$ & $\mathrm{~A}$ & $\mathrm{~T}$ \\
\hline & 121 & gtc & gga & $\operatorname{gcg}$ & act & gga & cac & cac & gtc & ctc & $\operatorname{ccg}$ & $g \operatorname{tg}$ & $\operatorname{ccg}$ & aca & gag & $g c c$ & gcc & $g g t$ & $g g c$ & $\operatorname{cct}$ & gca \\
\hline & & $\mathrm{V}$ & $\mathrm{G}$ & $\mathrm{A}$ & $\mathrm{T}$ & $\mathrm{G}$ & H & H & $\mathrm{V}$ & L & P & $\mathrm{V}$ & $\mathrm{P}$ & $\mathrm{T}$ & $\mathrm{E}$ & $\mathrm{A}$ & $\mathrm{A}$ & $\mathrm{G}$ & $\mathrm{G}$ & P & $\mathrm{A}$ \\
\hline & 181 & ttc & ttc & tca & tca & cac & ttc & aac & cac & tcc & tac & cac & cac & cac & caa & cag & acc & gce & cat & caa & aac \\
\hline & & $F$ & $F$ & 5 & 5 & $\mathrm{H}$ & $\mathrm{F}$ & $\mathbb{N}$ & $\mathrm{H}$ & $s$ & $\mathrm{Y}$ & $\mathrm{H}$ & $\mathrm{H}$ & $\mathrm{H}$ & $Q$ & $Q$ & $\mathrm{~T}$ & $\mathrm{~A}$ & $\mathrm{H}$ & $Q$ & $\mathrm{~N}$ \\
\hline & 241 & aac & ctc & tec & $\operatorname{cct}$ & t.tc & tat & gaa & tca & $\mathrm{cgc}$ & agc & tac & $g g c$ & act & gtg & $\operatorname{ccg}$ & tcc & gac & tac & cac & tct \\
\hline & & $\mathbf{N}$ & $\mathrm{I}$ & $\mathrm{F}$ & $\mathrm{P}$ & $\mathrm{F}$ & $Y$ & $\mathrm{E}$ & $s$ & $\mathrm{R}$ & $s$ & $Y$ & $\mathrm{G}$ & $T$ & $\mathrm{~V}$ & P & $\mathrm{s}$ & D & $Y$ & $\mathrm{H}$ & 5 \\
\hline & 301 & ggc & $\operatorname{cct}$ & gge & $\operatorname{ccc}$ & ttc & tac & cat & gac & $t c c$ & tac & $\mathrm{ccc}$ & $g g c$ & $\operatorname{ccg}$ & ggc & agc & gga & ggc & ctc & ttc & atg \\
\hline & & G & $\mathrm{P}$ & G & $\mathrm{P}$ & $\mathrm{F}$ & $Y$ & $\mathrm{H}$ & $\mathrm{D}$ & 5 & $\mathrm{Y}$ & $\mathrm{P}$ & G & $\mathrm{P}$ & G & 5 & $\mathrm{G}$ & $\mathrm{G}$ & $\mathrm{L}$ & $\mathrm{F}$ & $\mathbb{M}$ \\
\hline & 361 & $\operatorname{gcg}$ & $g t g$ & $\operatorname{ccg}$ & $\mathrm{aag}$ & agg & gag & gac & $\operatorname{gcg}$ & $\mathrm{gtg}$ & gtc & cag & $g c g$ & gca & cca & $g g t$ & $\operatorname{tcg}$ & $g g g$ & $g g c$ & gac & ttc \\
\hline & & $\mathbf{A}$ & $\mathrm{V}$ & $\mathrm{P}$ & $\mathrm{K}$ & $\mathrm{R}$ & $\mathrm{E}$ & $\mathrm{D}$ & $\mathrm{A}$ & $\mathrm{V}$ & $\mathrm{V}$ & $Q$ & $\mathrm{~A}$ & $\mathrm{~A}$ & P & $\mathrm{G}$ & 5 & $\mathrm{G}$ & $G$ & $\mathrm{D}$ & $\mathrm{F}$ \\
\hline & 421 & $\operatorname{acg}$ & gca & agg & att & ggg & $\operatorname{ctg}$ & aac & ctt & $\operatorname{ggg}$ & gge & cgt & $\mathrm{acg}$ & tac & ttc & tca & $\operatorname{tcg}$ & $g c g$ & gag & gac & gac \\
\hline & & $\mathrm{T}$ & A & $\mathbf{R}$ & I & $G$ & I & $\mathbb{N}$ & L & $G$ & $\mathrm{G}$ & $\mathrm{R}$ & $\mathrm{T}$ & $Y$ & F & $\mathrm{S}$ & $s$ & $\mathrm{~A}$ & $\mathrm{E}$ & D & $\mathrm{D}$ \\
\hline & 481 & ttc & gta & agc & $\operatorname{agg}$ & ctc & tac & $\operatorname{cgc}$ & $\operatorname{cgg}$ & $t c c$ & $\operatorname{cgg}$ & $\operatorname{ccg}$ & $\operatorname{ctg}$ & gag & gca & $g \operatorname{tg}$ & $g g g$ & $g g g$ & tct & gcc & $\mathrm{aag}$ \\
\hline & & $F$ & $\mathrm{~V}$ & $\mathrm{~s}$ & $\mathrm{R}$ & $\mathrm{L}$ & $Y$ & $\mathrm{R}$ & $\mathrm{R}$ & S & $\mathbb{R}$ & $\mathrm{P}$ & $\mathrm{L}$ & $\mathrm{E}$ & A & $\mathrm{V}$ & G & $\mathrm{G}$ & $\mathrm{s}$ & $\mathrm{A}$ & $\mathbb{K}$ \\
\hline & 541 & $\operatorname{gcg}$ & $\operatorname{ccg}$ & $\operatorname{cgg}$ & $\operatorname{tgc}$ & cag & $g c g$ & gag & agt & $\operatorname{tgc}$ & aac & $g \subset g$ & gac & $\operatorname{ctg}$ & $\mathrm{acg}$ & cac & $g c g$ & $\mathrm{aag}$ & cac & tac & cac \\
\hline & & $\mathrm{A}$ & $\mathrm{P}$ & $\mathrm{R}$ & C & $Q$ & $\mathrm{~A}$ & $\mathrm{E}$ & 5 & C & $\mathrm{N}$ & A & D & $\mathrm{L}$ & $\mathrm{T}$ & $\mathrm{H}$ & $\mathrm{A}$ & $\mathrm{K}$ & $\mathrm{H}$ & $Y$ & $\mathrm{H}$ \\
\hline & 601 & $\mathrm{cgg}$ & $\mathrm{cgg}$ & cac & $\mathrm{aag}$ & gtt & tgc & gag & ttc & cac & tcc & aag & gce & gce & gcc & gtc & ata & gct & gcc & $g g g$ & t.tg \\
\hline & & \begin{tabular}{|l}
$\mathrm{R}$ \\
\end{tabular} & $\mathrm{R}$ & $\mathrm{H}$ & $\mathrm{K}$ & $\mathrm{V}$ & C & $\mathrm{E}$ & $\bar{F}$ & $\mathrm{H}$ & 5 & $\mathrm{~K}$ & $\mathrm{~A}$ & $\mathrm{~A}$ & $\mathrm{~A}$ & $\mathrm{~V}$ & I & $\mathrm{A}$ & $\mathrm{A}$ & $\mathrm{G}$ & $\mathrm{L}$ \\
\hline & 661 & act & cag & cga & ttc & tgc & caa & cag & tgt & agc & agg & t.t & cac & ctt & ctc & $\operatorname{tcg}$ & gag & ttt & gac & aat & ggc \\
\hline & & $T$ & $Q$ & $\mathrm{R}$ & $F$ & C & $Q$ & $Q$ & $c$ & $s$ & $\mathrm{R}$ & $\mathrm{F}$ & $\mathrm{H}$ & I & I & $S$ & $\mathrm{E}$ & $F$ & $\mathrm{D}$ & $\mathrm{N}$ & $\mathrm{G}$ \\
\hline & 721 & aag & cga & agc & $\operatorname{tgc}$ & $\operatorname{cgg}$ & $\mathrm{aag}$ & $\mathrm{agg}$ & $\operatorname{ctg}$ & $g c c$ & gac & cat & $a a c$ & $\mathrm{cgc}$ & $\operatorname{cgc}$ & $\mathrm{cgg}$ & $a g g$ & aaa & tct & cag & caa \\
\hline & & $\mathrm{K}$ & $\mathrm{R}$ & 5 & C & $\mathrm{R}$ & $\mathrm{K}$ & $\mathrm{R}$ & $\mathrm{L}$ & $\mathbb{A}$ & $\mathrm{D}$ & $\mathrm{H}$ & $\mathrm{N}$ & $\mathrm{R}$ & $\mathrm{R}$ & $\mathrm{R}$ & $\mathrm{R}$ & $\mathrm{K}$ & $s$ & $Q$ & $Q$ \\
\hline & 781 & cag & $\mathrm{ccg}$ & aat & $\operatorname{ctg}$ & gaa & atc & cac & aag & cca & tct & cct & caa & gct & ggc & caa & agc & gca & $\operatorname{ttg}$ & att & gat \\
\hline & & $Q$ & $\mathrm{P}$ & $\mathbb{N}$ & $\mathrm{L}$ & $\mathrm{E}$ & I & $\mathrm{H}$ & $\mathrm{K}$ & $\mathrm{P}$ & 5 & P & $Q$ & $\mathrm{~A}$ & $\mathrm{G}$ & $Q$ & $s$ & $\mathbb{A}$ & $\mathrm{I}$ & I & $\mathrm{D}$ \\
\hline & 841 & agg & ctc & gca & atg & tct & $\operatorname{ctg}$ & cag & gat & $\operatorname{tcg}$ & ggg & aat & caa & tca & tca & tct & tcc & gtg & $a c c$ & ata & gca \\
\hline & & $\mathbb{R}$ & I & A & $\mathbb{M}$ & $s$ & L & $Q$ & D & $s$ & G & $\mathrm{N}$ & $Q$ & $s$ & $s$ & $\mathrm{~s}$ & $s$ & $\mathrm{~V}$ & $\mathrm{~T}$ & I & A \\
\hline & 901 & $g t g$ & tct & $\operatorname{cct}$ & $\operatorname{ccg}$ & aga & atg & $\operatorname{tcg}$ & ctc & gac & tct & tgt & ttc & $\operatorname{agg}$ & caa & aga & cca & ata & tat & aac & $\mathrm{cag}$ \\
\hline & & $\mathrm{V}$ & $\mathrm{s}$ & $\mathrm{P}$ & $\mathrm{P}$ & $\mathrm{R}$ & $M$ & $s$ & L & D & $\mathrm{s}$ & $c$ & $\mathrm{~F}$ & $\mathbb{R}$ & $Q$ & $\mathbb{R}$ & $\mathrm{P}$ & I & $Y$ & $\mathrm{~N}$ & $Q$ \\
\hline & 961 & caa & $\operatorname{ccc}$ & $\operatorname{cag}$ & gca & tct & tca & tet & tca & get & tca & tet & $g g c$ & tca & ctc & tet & ttc & tct & agc & $g g g$ & tga \\
\hline & & $Q$ & $\mathrm{P}$ & $Q$ & $\mathrm{~A}$ & 5 & 5 & 5 & 5 & $\mathrm{~A}$ & 5 & 5 & G & 5 & L & $F$ & $F$ & $s$ & 5 & $\mathrm{G}$ & - \\
\hline B & 1 & atg & gga & tca & aga & aga & $a a c$ & $\operatorname{ctc}$ & gaa & gat & cag & aag & $\mathrm{agg}$ & $\operatorname{agc}$ & ttc & $\operatorname{agg}$ & $g c g$ & gtc & gag & gag & gac \\
\hline & & $\mathrm{M}$ & G & S & $\mathrm{R}$ & $\mathrm{R}$ & $\mathrm{N}$ & $\mathrm{L}$ & $\mathrm{E}$ & D & $Q$ & $\mathrm{~K}$ & $\mathrm{R}$ & S & F & $\mathrm{R}$ & $\mathrm{A}$ & $\mathrm{V}$ & $\mathrm{E}$ & $\mathrm{E}$ & D \\
\hline & 61 & gag & gag & gac & gag & gag & gag & gag & gaa & gag & gag & gac & gag & gag & gag & gaa & gaa & gaa & gaa & tca & gag \\
\hline & & $\mathrm{E}$ & $E$ & D & $E$ & $E$ & $E$ & $\mathrm{E}$ & $E$ & $\mathrm{E}$ & $\mathrm{E}$ & D & $E$ & $E$ & $\mathrm{E}$ & $E$ & $\mathrm{E}$ & $\mathrm{E}$ & $E$ & S & $\mathrm{E}$ \\
\hline & 121 & agc & aaa & agg & ata & aga & gct & gct & ggt & gtg & agc & ttc & $\operatorname{tcg}$ & gca & $\operatorname{tcg}$ & $g g c$ & aag & aag & ttt & gga & tct \\
\hline & & $\mathrm{S}$ & $\mathrm{K}$ & $\mathrm{R}$ & I & $\mathrm{R}$ & A & $\mathrm{A}$ & G & V & $\mathrm{S}$ & $\mathrm{F}$ & $\mathrm{S}$ & $\mathrm{A}$ & $\mathrm{s}$ & G & $\mathrm{K}$ & $\mathrm{K}$ & $\mathrm{F}$ & G & $\mathrm{S}$ \\
\hline & 181 & tcc & $\operatorname{agc}$ & ggt & act & agt & gct & $g g c$ & $g g g$ & $\operatorname{tgg}$ & gct & $\operatorname{tcg}$ & $\mathrm{aca}$ & ggt & cga & $g \subset g$ & $\mathrm{acc}$ & act & $\operatorname{tgc}$ & cag & $\mathrm{gcc}$ \\
\hline & & $S$ & $S$ & G & $\mathrm{T}$ & $S$ & $\mathrm{~A}$ & G & G & W & A & $\mathrm{S}$ & $\mathrm{T}$ & G & $\mathrm{R}$ & $\mathrm{A}$ & $\mathrm{T}$ & $\mathrm{T}$ & $\mathrm{C}$ & $Q$ & $\mathrm{~A}$ \\
\hline & 241 & gac & $\mathrm{aac}$ & $\operatorname{tgc}$ & $\mathrm{acg}$ & $\mathrm{acc}$ & gat & atg & act & gag & $g c c$ & aag & $\mathrm{cgg}$ & tac & $\mathrm{cac}$ & cga & $\operatorname{cgc}$ & $\mathrm{cac}$ & aag & gtg & $\operatorname{tgt}$ \\
\hline & & D & $\mathrm{N}$ & $\mathrm{C}$ & $\mathrm{T}$ & $\mathrm{T}$ & $\mathrm{D}$ & $\mathrm{M}$ & $\mathrm{T}$ & $E$ & A & $\mathrm{K}$ & $\mathrm{R}$ & $Y$ & $\mathrm{H}$ & $\mathrm{R}$ & $\mathrm{R}$ & $\mathrm{H}$ & $\mathrm{K}$ & $\mathrm{V}$ & $\mathrm{C}$ \\
\hline & 301 & gag & ttc & cat & $g c c$ & aag & $g c c$ & cag & gtg & gtc & ctc & att & gac & $g g g$ & atc & $\mathrm{cac}$ & cag & $\operatorname{cgc}$ & ttc & $\operatorname{tgt}$ & cag \\
\hline & & $E$ & $F$ & $\mathrm{H}$ & $\mathrm{A}$ & $\mathrm{K}$ & A & Q & $\mathrm{V}$ & $\mathrm{V}$ & $\mathrm{I}$ & I & D & $\mathrm{G}$ & I & $\mathrm{H}$ & $Q$ & $\mathrm{R}$ & $F$ & C & $Q$ \\
\hline & 361 & caa & $\operatorname{tg} c$ & agc & agg & ttc & cat & gag & ctt & $\mathrm{acg}$ & gag & ttc & gat & gaa & $\operatorname{gcg}$ & aaa & aga & $\operatorname{agc}$ & $\operatorname{tgc}$ & $\mathrm{cgg}$ & aga \\
\hline & & $Q$ & $\mathrm{C}$ & $S$ & $R$ & $\mathrm{~F}$ & $\mathrm{H}$ & $E$ & $\mathrm{~L}$ & $\mathrm{~T}$ & $E$ & $F$ & $\mathrm{D}$ & $E$ & $\mathrm{~A}$ & $\mathrm{~K}$ & $R$ & $S$ & $\mathrm{C}$ & $R$ & $\mathrm{R}$ \\
\hline & 421 & $\operatorname{agg}$ & $\operatorname{ttg}$ & get & ggt & $\mathrm{cac}$ & $\mathrm{aac}$ & gag & $\operatorname{cgg}$ & cgt & cga & aag & agc & $\operatorname{tcg}$ & tac & gat & tet & cct & gga & gaa & ggc \\
\hline & & $\mathrm{R}$ & $\mathrm{L}$ & $\mathrm{A}$ & $\mathrm{G}$ & $\mathrm{H}$ & $\mathrm{N}$ & $E$ & $R$ & $R$ & $R$ & $\mathrm{~K}$ & $\mathrm{~S}$ & S & $\mathrm{Y}$ & $\mathrm{D}$ & $\mathrm{F}$ & P & G & $\mathrm{E}$ & G \\
\hline & 481 & tca & aac & tga & & & & & & & & & & & & & & & & & \\
\hline & & $S$ & $\mathrm{~N}$ & - & & & & & & & & & & & & & & & & & \\
\hline
\end{tabular}




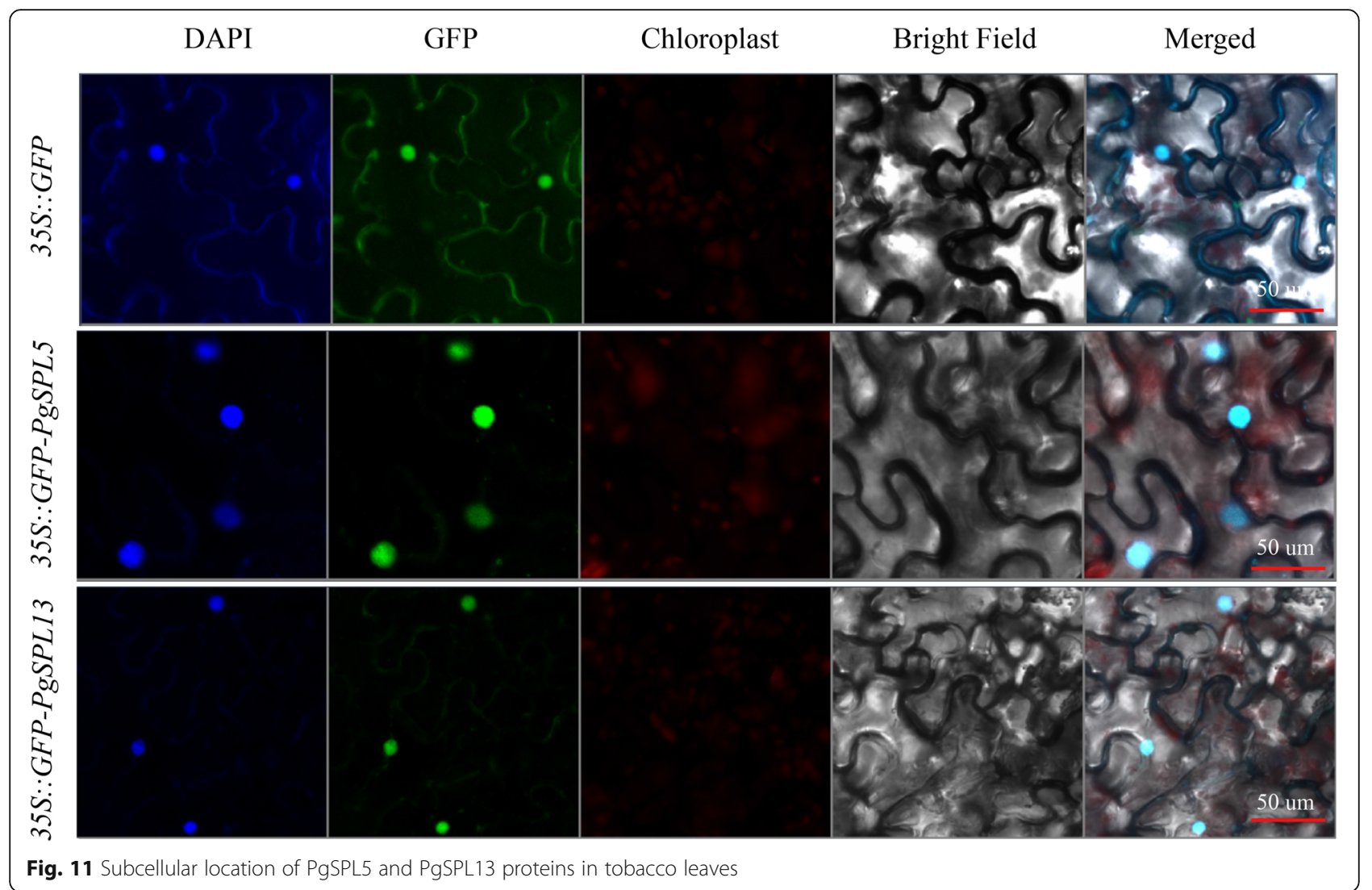

the expression of PgSPL13 presented the same changes in leaves and stems after IBA treatment as 6-BA treatment.

We can see from Fig. 12e and $\mathrm{f}$, the expression of PgSPL5 and PgSPL13 were significantly up-regulated in P2 stage of functional male flowers after spraying exogenous PP333, while the expression of these two genes was inhibited in other stages of flower development. PgSPL5 was up-regulated in leaves and stems, while PgSPL13 was up-regulated in leaves but down-regulated in stems. This is consistent with the trend observed within 6-BA and IBA treatments.

\section{Discussion}

In recent years, many studies have pointed out that the SPL transcription factor family play fundamental roles in plant growth and development and governing a variety of physiological processes $[19,46,47]$. In this study, a total of 15 SPL genes were identified from the whole genome of 'Taishanhong' pomegranate using bioinformatics methods. The information we obtained from the phylogenetic analysis was that these 15 SPL genes were clustered into six subgroups. This result indicated that $P$. granatum, $A$. thaliana, $M$. domestica and Vitis vinifera were relatively conserved during the evolution [48]. Gene structure and motif composition analyses showed that PgSPL genes within the same group shared similar motifs and exonintron organization, suggesting that these genes may derived from the same ancestral gene and perform similar functions in plant growth and development [49]. Compared with other subgroups, there are many more complex conserved domains in subgroups G1 and G6, and members of these groups may have other functions [50].

The role of cis-acting elements influencing the gene expression is gaining emphasis in recent times [2]. This study found that all PgSPL promoters contained multiple cis-acting elements associated with abiotic stress or hormonal response, suggesting that PgSPL may play functions in plant response to abiotic stress and hormonal stress. In particular, the discovery of hormoneinduced related elements such as auxin and gibberellin suggested that PgSPL family genes were related to the growth and development of pomegranate.

SPL family genes are regulated by miRNA156. There are 11 SPL genes in Arabidopsis that are regulated by miR156, which play multiple functions in plant growth and development $[29,30,32]$. This study predicted that in pomegranate, of the 15 SPL family members, 10 possess binding sites for miR156. In addition to the potential miR156 targets of PgSPL2, PgSPL13 and PgSPL14 in G5 subgroup were located at 3'UTR, the potential targets of other genes were located in the coding region. 

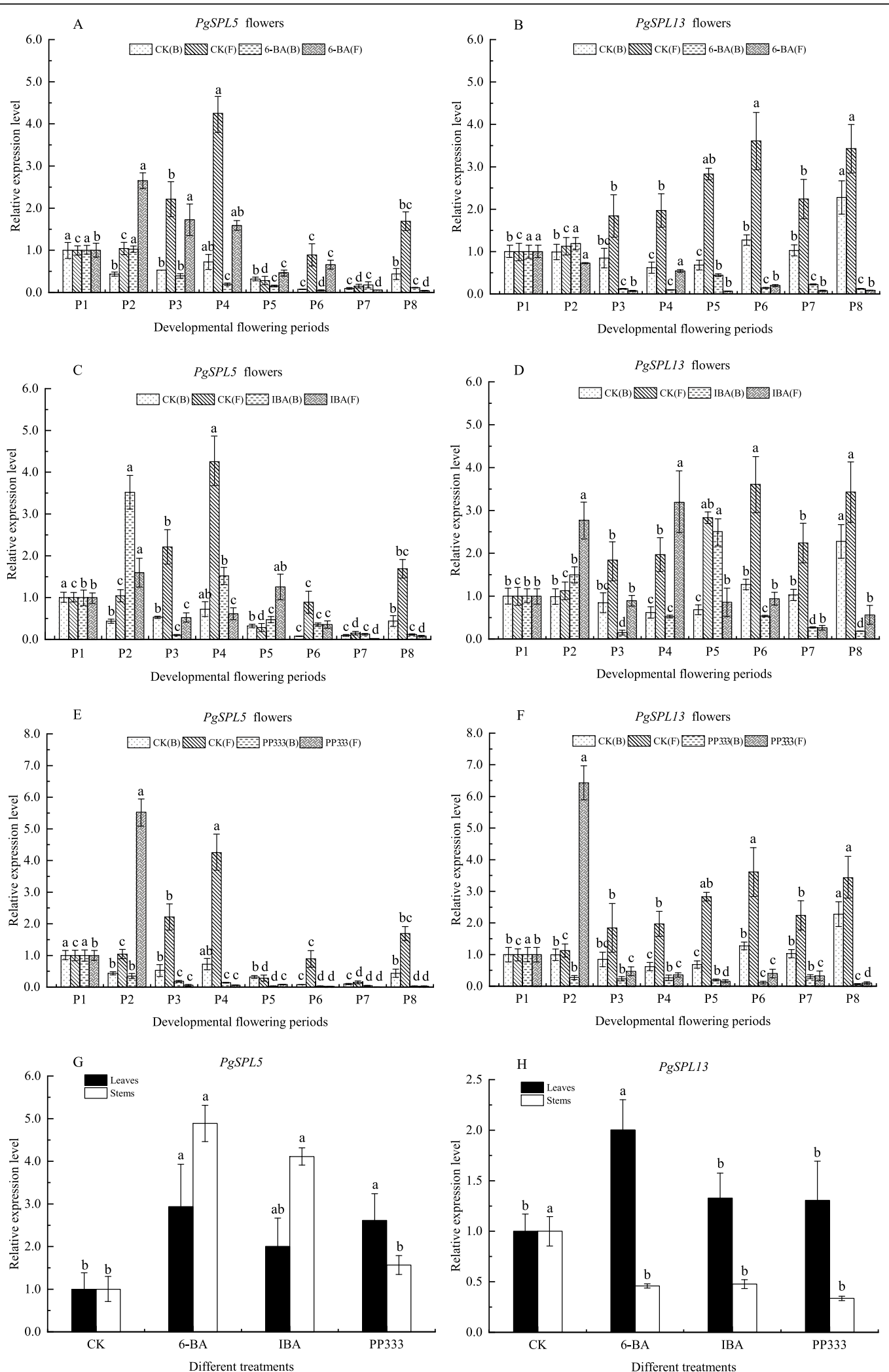

Fig. 12 (See legend on next page.) 
(See figure on previous page.)

Fig. 12 Expression levels of PgSPL5 and PgSPL13 following different treatments in different organizations. a-h are the expression levels of PgSPL5 and PgSPL13 in bisexual flowers, functional male flowers, leaves and stems, respectively. In the illustration (B) and (F) represent bisexual flowers and functional male flowers respectively. The vertical bar in bar graph is standard error. Bars with different letters within each panel are significant differences at $p<0.05$ according to the Turkey's test

Transcriptome expression data showed that most PgSPL genes are expressed in leaves, roots, flowers, seed coats amd pericarps, except that very few PgSPLs are not expressed in a mixture of pericarp and various tissues. This indicates that the PgSPL family genes are involved in pomegranate leaf development, phase transition, floral development and fruit ripening and other processes. Most PgSPL genes were highly expressed in differently developmental periods of bisexual flowers and functional male flowers, these data support the hypothesis that PgSPL genes play an important role in pomegranate flowering. At the same time, it was found that the expression of PgSPL4 in various tissues was not significantly different, which may be related to the mutation of the $\mathrm{C} 3 \mathrm{H}$ region of the zinc finger structure of the gene encoding protein that affected its gene function. PgSPL8, PgSPL9 and PgSPL10 are generally highly expressed in all tissues, which may be related to the existence of other more conserved motifs. Additionally, the level of PgSPL15 was relatively low in all tissues. Therefore, it can be presumed that its role in regulating pomegranate development was weak. We also analyzed the expression differences of miR156a-5p and PgSPL13 during the three different stages of pomegranate bisexual flower and functional male flower development, it was found that their expression levels showed an opposite tendency, which was consistent with the conclusion that miR156 negatively regulated the expression of its target genes [29].

Eight PgSPLs with significant differences in expression in different tissues of pomegranate were analyzed by qRT-PCR. The results showed that the expression levels of PgSPL2, PgSPL13 and PgSPL14 in functional male flowers were higher than those in bisexual flowers from P1 to P8 stages, showing a similar expression pattern. The expression patterns of PgSPL6 and PgSPL11 in the two flowers were also similar, which may be related to their clustering in the same subgroup and playing similar functions [50]. Part of flower bud pistil abortion during development of pomegranate to form functional male flowers, and functional male flower pistil abortion is due to abnormal ovule development, can not form egg cells [51-53]. Studies have shown that flower bud longitudinal diameter $5.1 \sim 15.0 \mathrm{~mm}$ is an important stage of pomegranate ovule development [54]. PgSPL5 had higher expression in functional male flowers than in bisexual flowers. At P2 P4 $(5.1 \sim 12.0 \mathrm{~mm})$ stages of functional male flowers, the expression levels of PgSPL5 were significantly increased. Therefore, we speculated that PgSPL5 may be involved in the regulation of functional male flower formation in pomegranate. PgSPL12 showed similar expression patterns in the two types of flowers before ovule development abnormality, and it mainly performed functions in functional male flowers after ovule development abnormality. PgSPL12, AtSPL9 and AtSPL15 were clustered in the same branch, while AtSPL9 and AtSPL15 could regulate flowering time [28] and induce Arabidopsis flowering [30]. This indicated that PgSPL12 also played an important role in flower development. The expression levels of PgSPL13 were relatively stable in two types of flower development, which indicated that PgSPL13 played a role in pomegranate flower organ development. We also found that the expression levels of PgSPL2, PgSPL3, PgSPL6, PgSPL11 and PgSPL14 in young leaves were significantly higher than those in buds and stems. This suggested that these genes played important roles in leaves development. The expression levels of PgSPL5, PgSPL12 and PgSPL13 in flower buds were significantly higher than that in leaves and stems, which further indicated that these three genes had important functions in the development of pomegranate flowers.

In Arabidopsis, some AtSPL genes such as SPL3 and $S P L 8$ are involved in the response to GA signal transduction in plant development such as flowering transformation [34] and anther development [39]. Many studies have shown that SPL3 has a very important function in the development of Arabidopsis flowers [35, 38, 55]. In addition, SPL3, SPL4 and SPL5 are involved in the control of flowering time and developmental transition of Arabidopsis [56]. Studies have found that homologs from different species usually share a similar function in the biological process. For example, the orthologous genes OsSPL13, ZmSBP6 and AmSBP1 of $S P L 3$ are involved in inflorescence and flower in $O$. sativa [13], Zea mays [57] and A. majus [58], respectively. In this study, we cloned SPL8 and SPL3 homologous genes PgSPL5 and PgSPL13 from 'Taishanhong' by homologous cloning technology, and the subcellular localization verification proved that they performed their functions in the nucleus. We studied their spatiotemporal expression characteristics under the treatment of exogenous hormones, and found that after exogenous hormone treatment, the expression of PgSPL5 and PgSPL13 were up-regulated or down-regulated by different plant hormones. They were differentially expressed 
in leaves, stems, bisexual flowers, functional male flowers and other tissues of pomegranate, and each of them respond differently to various different hormones. It indicates that these two genes may be involved in the response process of different plant hormone signal transduction in pomegranate development. We found that in flower tissues, this response was stronger when the buds were young (P2 stage), and the levels of these two genes were significantly affected by the 6-BA treatment in leaves and stems. This study only analyzed the possible functions of the two genes PgSPL5 and PgSPL13 in the development of pomegranate. However, how these two genes perform their functions and whether there is a mechanism between them remain to be further studied.

\section{Conclusions}

In this study, we identified and characterized 15 SPL genes in pomegranate. Phylogenetic analysis indicated that the PgSPLs were divided into six subgroups, with a similar distribution of conserved motifs and exon-intron organization in the members of each subgroup. And there are 10 SPL genes in pomegranate that are regulated by miR156. Based on the transcriptome data of different tissues, we found certain PgSPL genes played significant roles in pomegranate growth and development. qRT-PCR analysis showed that PgSPL2, PgSPL3, PgSPL6, PgSPL11 and PgSPL14 played roles in leaves development of pomegranate. PgSPL5, PgSPL12 and PgSPL13 played roles in pomegranate flower development. The subcellular localization results showed that PgSPL5 and PgSPL13 proteins were located in the nucleus. Furthermore, exogenous plant growth regulator induction experiments showed that PgSPL5 and PgSPL13 were involved in the response process of different plant hormone signal transduction in pomegranate development. And we found that in flower tissues, this response was stronger when the buds were young (P2 stage), and the levels of these two genes were significantly affected by the 6-BA treatment in leaves and stems.

\section{Methods}

\section{Identification and sequence analysis of SPL gene family}

The HMM profile of the Pfam SBP/SPL domain (PF03110) [8] was performed against 'Taishanhong' pomegranate protein databases using HMMER 3.0 (http://hmmer.org/) software package (Evalue $<1 \mathrm{e}^{-5}$ ) [1], duplication was removed and SPL protein sequences were screened and selected. SPL protein sequences of $A$. thaliana, E. grandis, M. domestica and Vitis vinifera in PlantTFDB database (http://planttfdb.gao-lab.org/index. php) [59] were used as queries to perform BLAST against the pomegranate genome database (E-value < $1 \mathrm{e}^{-5}$, identity $>50 \%$ ) [3]. Moreover, all obtained SPL protein sequences were further analyzed on CDD website (https://www.ncbi.nlm.nih.gov/cdd) [60], and SMART (http://smart.embl-heidelberg.de/) [61] to verify the conserved domains of SBP domain.

The online tool ExPaSy-Protparam (https://web. expasy.org/protparam/) [62] was used to predict and analyze the physical and chemical properties of pomegranate SPL family, such as molecular weight and isoelectric point. Subcellular localization was predicted using the online website (http://www.csbio.sjtu.edu.cn/ bioinf/Cell-PLoc-2/) [45].

\section{Phylogenetic analysis}

In order to explore the phylogenetic relationship of pomegranate SPL family genes, SPL proteins of $A$. thaliana (17), M. domestica (27) and Vitis vinifera (18) were downloaded for multiple sequences alignment. An unrooted Neighbor-Joining (NJ) phylogenetic tree was constructed with all of SPL protein sequences using MEGA 7.0, bootstrap $=1000$ repetitions, Complete deletion, and Poisson model [63]. EvolView (https://www. evolgenius.info/evolview/) [64] online was used to beautify the phylogenetic tree.

\section{Gene structure analysis and motif identification}

Motifs of the SPL proteins were identified using MEME online tools (http://meme-suite.org/) [65] with default parameter, and the motif characteristics of the SPL proteins were obtained. The exon-intron structures of the $S P L$ genes were determined based on the annotation information of 'Taishanhong' genome, then the results were displayed using GSDS 2.0 (http://gsds.cbi.pku.edu. cn) online tools [66].

\section{Cis-acting elements}

The $1500 \mathrm{bp}$ upstream sequence of the initial codon were obtained from pomegranate genome sequence for cis-acting element analysis by the PlantCARE (http://bioinformatics.psb.ugent.be/webtools/plantcare/ $\mathrm{html} /$ ) [67].

\section{Prediction of PgSPL genes targeted by miR156}

Using psRNATarget online software (http://plantgrn. noble.org/psRNATarget/) [68] to analyze the nucleotide sequence of pomegranate SPL genes and predict the target gene site of pomegranate miR156. DNAMAN software was used to perform multiple sequence alignment between the reverse complement of PgmiR156 and PgSPL sequences [43].

\section{Expression patterns by transcriptome data}

To examine expression patterns of SPL genes in different pomegranate tissues and organs, the published transcriptome data were download for expression analysis. The 
transcript data of bisexual flowers, functional male flowers, leaf, root, outer seed coat, inner seed coat, and pericarp of pomegranate were obtained from NCBI database (http://www.ncbi.nlm.nih.gov/) [3]. The registration numbers are 'Dabenzi' SRR5279388, SRR5279391, SRR5279394-SRR5279397; 'Tunisia' SRR5446592, SRR5446595, SRR5446598, SRR5446601, SRR5446604, SRR5446607 and SRR5678820; 'Baiyushizi' SRR5678819; 'Black127' SRR1054190; 'nana' SRR1055290 and 'Wonderful' SRR080723. Firstly, all RNA-Seq data were qualitatively controlled by fastp to obtain cleaned reads. Then the sequence was indexed with pomegranate transcriptome data. Through Kallisto 0.44.0 software [69], and the gene expression was further calculated and analyzed. The corresponding expression level (TPM value) of SPL genes was thus obtained. TPM value was transformed into $\log _{2}(\mathrm{TPM}+1)$. Finally, the heat map of PgSPL was drawn with TBtools [1].

At the same time, the expression of miR156 of pomegranate bisexual flowers and functional male flowers at three different stages (bud vertical diameter of buds were $3.0 \sim 5.0 \mathrm{~mm}, 5.1 \sim 13.0 \mathrm{~mm}, 13.0 \sim 20.0$ $\mathrm{mm})$ was analyzed.

\section{Plant material and exogenous hormone treatments}

Research conducted at Baima Base for Teaching and Scientific Research of Nanjing Forestry University. The tested cultivars were 6-year-old 'Taishanhong' pomegranate. Three plant growth regulators were selected and the spraying concentration was as follows (Table 2) [70]. Each group consisted of 3 plants, and each was sprayed to drip. Individual plants were treated on October 15, 2019 (dormancy period), April 15, 2020 (spring leaf expansion period), May 10 (initial flowering to full flowering period) and May 25 (end of full flowering period), respectively.

No permission is required for sample collection. New leaves and stems were collected after 7 days of each treatment from May 10, and the bisexual flowers and functional male flowers at different developmental stages of pomegranate were collected. Referring to Chen and Zhao's study on the morphological and embryological differences of pomegranate flowers $[3,71]$, the flower buds were divided into eight stages according to their longitudinal diameter (Fig. 13): $3.0 \sim 5.0 \mathrm{~mm}$ (P1), 5.1

Table 2 Treatment types and concentrations

\begin{tabular}{ll}
\hline Types of treatment & Concentrations $(\mathbf{m g} / \mathbf{L})$ \\
\hline $6-B A$ & 100 \\
IBA & 20 \\
PP333 & 1000 \\
CK & Water \\
\hline
\end{tabular}

6-BA 6-Benzylaminopurine, IBA Indole-3-butyric acid, PP333 Paclobutrazol
$8.0 \mathrm{~mm}$ (P2), $8.1 \sim 10.0 \mathrm{~mm}$ (P3), $10.1 \sim 12.0 \mathrm{~mm} \mathrm{(P4),}$ $12.1 \sim 14.0 \mathrm{~mm} \quad$ (P5), $14.1 \sim 16.0 \mathrm{~mm} \quad$ (P6), $16.1 \sim 18.0$ $\mathrm{mm}$ (P7), and $18.1 \sim 20.0 \mathrm{~mm}$ (P8). Samples were frozen with liquid nitrogen and stored in $-78^{\circ} \mathrm{C}$ refrigerator for reserve.

\section{RNA isolation and gene cloning}

Total RNA was extracted using the BioTeke plant total RNA extraction kit (BioTeke Corporation, Beijing, China), and first-strand cDNA was prepared using a reverse transcription kit-PrimeScript ${ }^{\text {six }}$ RT reagent Kit with gDNA Eraser (TaKaRa Bio Tech Co., Ltd., Beijing, China). Then the cDNA was used as template for gene clone and expression analysis on SPL family genes at different developmental stages of pomegranate. According to DNA and CDS sequences, specific quantitative primers (Table 3) were designed using Oligo 7 software [72].

Each $50 \mu \mathrm{L}$ of reaction mixture contained $25 \mu \mathrm{l} 2 \times$ Taq Plus Master Mix, $1 \mu \mathrm{l}$ each of the upstream/downstream primers, $2 \mu \mathrm{l}$ of cDNA template and $21 \mu \mathrm{l}$ Nuclease-free ddH2O. PCR program was set as follows: predenaturation at $95^{\circ} \mathrm{C}$ for $3 \mathrm{~min} ; 95^{\circ} \mathrm{C}$ for $15 \mathrm{~s}, 58^{\circ} \mathrm{C}$ for $45 \mathrm{~s}, 72^{\circ} \mathrm{C}$ for $1 \mathrm{~min}$, a total of 35 cycles; $72^{\circ} \mathrm{C}$ for $5 \mathrm{~min}$. Firstly, the PCR products were separated by $1 \%$ agarose gel electrophoresis, and the target fragment was recovered by gel cutting. The recombinant plasmid was connected to the overexpression vector, and transformed into competent E. coli DH5 $\alpha$. Then, the positive monoclonal detected by PCR was selected and sent to Shanghai Bioengineering Company for sequence. Finally, the sequences obtained by sequencing were translated into amino acid sequences using ExPaSy-Translate online tool (https://web.expasy.org/translate/) [62].

\section{Subcellular location analyses}

To verify the location of PgSPL5 and PgSPL13, the fulllength coding sequence (CDS) without stop codon was cloned into the pBI121 and C-terminal fused with the green fluorescent protein (GFP). Primers for the restriction sites at both ends of the target gene were designed (Table 3). The product was recovered from the recombination ligation gel, and the recombinant plasmid obtained from the ligation was introduced into Agrobacterium GV3101 by freeze-thaw method. The vector GV3101 (A. tumefaciens) harbouring 35S::GFP-PgSPL and control vector were infiltrated into tobacco (Nicotiana benthamiana) leaves. After $36 \sim 48 \mathrm{~h}$, Fluorescence images were captured using an LSM 710confocal laserscanning microscope (Zeiss, Jena, Germany) [72, 73].

Real-time fluorescent quantitative PCR analysis (qRT-PCR) The expression of PgSPLs in different tissues was analyzed by qRT-PCR, and the expression of PgSPL5 and PgSPL13 under different growth regulators was analyzed. 


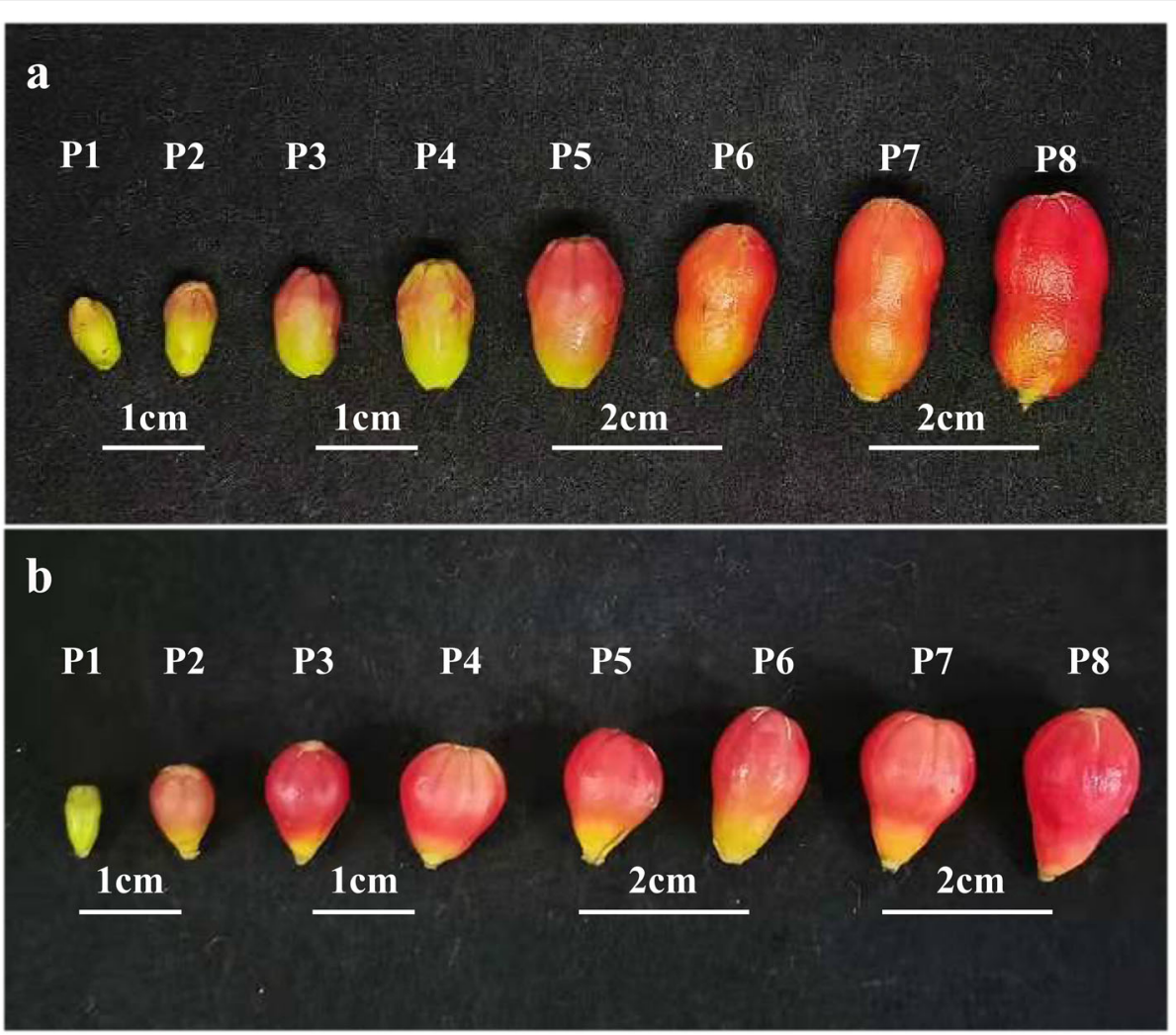

Fig. 13 Bisexual flower (a) and Functional male flower (b) at different developmental stages (CK)

Table 3 Primers used in this study

\begin{tabular}{|c|c|c|}
\hline Primer & Primer sequence $\left(5^{\prime}-3^{\prime}\right)$ & Annotation \\
\hline PgSPL5 & $\begin{array}{l}\text { F: ATGTTGGACTATGAATGGGC } \\
\text { R: TCACCCGCTAGAGAAAAAGAG }\end{array}$ & Gene clone \\
\hline PgSPL13 & $\begin{array}{l}\text { F: ATGGGATCAAGAAGAAACCTCGAAG } \\
\text { R: TCAGTTTAGCCTTCTCCAGGAAAA }\end{array}$ & Gene clone \\
\hline GFP-PgSPL5 & $\begin{array}{l}\text { F:gagaacacgggggactctagaATGTTGGACTATGAATGGGC } \\
\text { R:gcccttgctcaccatggatccTCACCCGCTAGAGAAAAAGAG }\end{array}$ & subcellular localization \\
\hline GFP-PgSPL13 & $\begin{array}{l}\text { F:gagaacacgggggactctagaATGGGATCAAGAAGAAACCTCGAAG } \\
\text { R:gcccttgctcaccatggatcCTCAGTTGAGCCTTCTCCAGGAAAA }\end{array}$ & subcellular localization \\
\hline qRT-PgSPL2 & $\begin{array}{l}\text { F:AAGAGAGACTTCACCACCGAG } \\
\text { R: GTGAAACCTGCTGCATTGCT }\end{array}$ & Gene expression \\
\hline qRT-PgSPL3 & $\begin{array}{l}\text { F: AACAGCCGAGACTCATCCC } \\
\text { R: GAGGTCCTTGTACACCCGTA }\end{array}$ & Gene expression \\
\hline qRT-PgSPL5 & $\begin{array}{l}\text { F: GAAATCTCAGCAACAGCCGAAT } \\
\text { R: CTITGCCTGAAACAAGAGTCG }\end{array}$ & Gene expression \\
\hline qRT-PgSPL6 & $\begin{array}{l}\text { F: AATGCAGCAGGTTTCATTCCC } \\
\text { R: TCGTITICCGACTTGACAGC }\end{array}$ & Gene expression \\
\hline qRT-PgSPL11 & $\begin{array}{l}\text { F: TTCCCAGCCATTCCATCCTCG } \\
\text { R: GGCTTCGCTATACCAAGTCCT }\end{array}$ & Gene expression \\
\hline qRT-PgSPL12 & $\begin{array}{l}\text { F: AAGTCCCCTAAAGTCATCGTT } \\
\text { R: TGAAGTCCATCACAAAGCCT }\end{array}$ & Gene expression \\
\hline qRT-PgSPL13 & $\begin{array}{l}\text { F: CGACAACTGCACGACCGAT } \\
\text { R: CGGCAGCTTCTITCGCTTC }\end{array}$ & Gene expression \\
\hline qRT-PgSPL14 & $\begin{array}{l}\text { F: TCGACGATACCAAGAGAAGCTG } \\
\text { R: AATTCTCCCCCGATCATCGAC }\end{array}$ & Gene expression \\
\hline PgActin & $\begin{array}{l}\text { F: AGTCCTCTTCCAGCCATCTC } \\
\text { R: CACTGAGCACAATGTITCCA }\end{array}$ & Gene expression \\
\hline
\end{tabular}


Specific quantitative primers (Table 3) were designed, and pomegranate PgActin gene was used as an internal reference. Each $20 \mu \mathrm{L}$ of reaction mixture contained $10 \mu \mathrm{L}$ of TB Green Premix Ex Taq, 0.4 $\mu \mathrm{L}$ of ROX Reference Dye II, $0.4 \mu \mathrm{L}$ of upstream/downstream primers, $2 \mu \mathrm{L}$ of cDNA template and $6.8 \mu \mathrm{L}$ of ddH2O. qRT-PCR was performed using a 7500 fast Real-Time PCR system (Applied Biosystems, CA, USA) with three biological and three technical replicates for each cDNA sample. The thermal cycler was set as follows: pre-denaturation at $95^{\circ} \mathrm{C}$ for $30 \mathrm{~s}, 40$ cycles of $95^{\circ} \mathrm{C}$ for $5 \mathrm{~s}$ and $60^{\circ} \mathrm{C}$ for $34 \mathrm{~s}$; the dissociation stage was set as follows: $95^{\circ} \mathrm{C}$ for $15 \mathrm{~s}, 60^{\circ} \mathrm{C}$ for $60 \mathrm{~s}$ and $95^{\circ} \mathrm{C}$ for $15 \mathrm{~s}$. The data was quantitatively analyzed by the $2^{-\Delta \Delta \mathrm{Ct}}$ method [74].

\section{Data analysis}

All data of qRT-PCR were analyzed with one-way ANOVA, and multiple comparisons were evaluated with the Turkey's test $(p<0.05)$ using the SPSS program (Version 19.0. Chicago, IL, USA) based on the values of three complete randomized blocks.

\begin{abstract}
Abbreviations
SPL: SQUAMOSA promoter-binding protein-like; A. thaliana: Arabidopsis thaliana; E. grandis: Eucalyptus grandis; IBA: Indole-3-butyric acid; 6-BA: 6Benzylaminopurine; PP333: Paclobutrazol; RNA: Ribonucleic Acid; CDNA: complementary DNA; qRT-PCR: Quantitative real-time polymerase chain reaction
\end{abstract}

\section{Supplementary Information}

The online version contains supplementary material available at https://doi. org/10.1186/s12870-021-03171-7.

\section{Additional file 1.}

\section{Acknowledgements}

Not applicable.

\begin{abstract}
Authors' contributions
$B L$ and $Y Z$ participated in the experimental design, and also analyzed and interpreted the phylogenetic analysis; BL and SW performed the expression level examination of the plants; BL, YS and YW contributed reagents and analysis tools; BL was a major contributor in experimentation, analyzing data and writing the manuscript; $Y Z, X Z$ and $Z Y$ revised the manuscript. All authors read and approved the final manuscript.

\section{Funding}

This work was supported by the Initiative Project for Talents of Nanjing Forestry University (GXL2014070, GXL2018032), the Doctorate Fellowship Foundation of Nanjing Forestry University, and the Priority Academic Program Development of Jiangsu High Education Institutions (PAPD), the National Natural Science Foundation of China (31901341), the Natural Science Foundation of Jiangsu Province (BK20180768). These funding bodies took part in the design of the study and collection, analysis, and interpretation of data, and the writing of the manuscript, as well as in the open access payment.
\end{abstract}

\section{Availability of data and materials}

The whole genome data of pomegranate is downloaded from the NCBI database (https://www.ncbi.nlm.nih.gov/search/all/?term=ASM220158v1), and the accession number is ASM220158v1. The transcriptome data is obtained from NCBI (https://www.ncbi.nlm.nih.gov/Traces/study/), and the accession numbers are SRP103147 and SRP100581. The SPL protein sequences of $A$. thaliana, E. grandis, M. domestica and Vitis vinifera are downloaded from the PlantTFDB database (http://planttfdb.gao-lab.org/index.php). Public access to all databases is open. The datasets supporting the conclusions of this article are included within the article (and its additional files).

\section{Declarations}

Ethics approval and consent to participate

Not applicable.

\section{Consent for publication}

Not applicable.

\section{Competing interests}

The authors declare that they have no competing interests.

Received: 18 March 2021 Accepted: 10 August 2021

Published online: 28 August 2021

\section{References}

1. Zhao Y, Zhao H, Wang Y, Zhang X, Zhao X, Yuan Z. Genome-wide identification and expression analysis of MIKC-type MADS-box gene family in Punica granatum L. Agronomy. 2020;10(8):1197.

2. Wang Y, Zhao Y, Yan M, Zhao H, Zhang X, Yuan Z. Genome-wide identification and expression analysis of TALE gene family in Pomegranate (Punica granatum L.). Agronomy. 2020:10(6):829.

3. Zhao Y, Liu C, Ge D, Yan M, Ren Y, Huang X, et al. Genome-wide identification and expression of YABBY genes family during flower development in Punica granatum L. Gene. 2020;752:144784. https://doi. org/10.1016/j.gene.2020.144784.

4. Zhu T, Liu Y, Ma L, Wang X, Zhang D, Han Y, et al. Genome-wide identification, phylogeny and expression analysis of the SPL gene family in wheat. Plant Biol. 2020;20(1):420. https://doi.org/10.1186/s12870-02002576-0.

5. Preston JC, Hileman LC. Functional evolution in the plant SQUAMOSAPROMOTER BINDING PROTEIN-LIKE (SPL) gene family. Front Plant SCi. 2013;4:80.

6. Rhoades MW, Reinhart BJ, Lim LP, Burge CB, Bartel B, Bartel DP. Prediction of plant microRNA targets. Cell. 2002;110(4):513-20. https://doi.org/10.1016/ S0092-8674(02)00863-2.

7. Yamasaki K, Kigawa T, Inoue M, Tateno M, Yamasaki T, Yabuki T, et al. A novel zincbinding motif revealed by solution structures of DNA-binding domains of Arabidopsis SBP-family transcription factors. J Mol Biol. 2004; 337(1):49-63. https://doi.org/10.1016/j.jmb.2004.01.015.

8. Birkenbihl RP, Jach G, Saedler H, Huijser P. Functional dissection of the plant-specific SBP-domain: overlap of the DNA binding and nuclear localization domains. J Mol Biol. 2005;352(3):585-96. https://doi.org/10.1016/ j.jmb.2005.07.013.

9. Klein J, Saedler H, Huijser P. A new family of DNA binding proteins includes putative transcriptional regulators of the Antirrhinum majus floral meristem identity gene SQUAMOSA. Mol Gen Genomics. 1996;250(1):7-16.

10. Cardon G, Höhmann S, Klein J, Nettesheim K, Saedler H, Huijser P. Molecular characterization of the Arabidopsis SBP-box genes. Gene. 1999;237(1):91-104. https://doi.org/10.1016/S0378-1119(99)00308-X.

11. Li C, Lu S. Molecular characterization of the SPL gene family in Populus trichocarpa. BMC Plant Biol. 2014;14(1):131. https://doi.org/10.1186/1471-222 9-14-131.

12. Salinas M, Xing S, Höhmann S, Berndtgen R, Huijser P. Genomic organization, phylogenetic comparison and differential expression of the SBP-box family of transcription factors in tomato. Planta. 2012;235(6):117184. https://doi.org/10.1007/s00425-011-1565-y.

13. Xie K, Wu C, Xiong L. Genomic organization, differential expression, and interaction of SQUAMOSA promoter-binding-like transcription factors and microRNA156 in rice. Plant Physiol. 2006;142(1):280-93. https://doi.org/10.11 04/pp. 106.084475

14. Li J, Hou H, Li X, Xiang J, Yin X, Gao H, et al. Genome-wide identification and analysis of the SBP-box family genes in apple (Malus $\times$ domestica Borkh.). Plant Physiol Biochem. 2013;70:100-14. https://doi.org/10.1016/j.pla phy.2013.05.021.

15. Hou H, Li J, Gao M, Singer S, Wang H, Mao L, et al. Genomic organization, phylogenetic comparison and differential expression of the SBP-box family 
genes in grape. PLoS One. 2013;8(3):e59358. https://doi.org/10.1371/journal. pone.0059358.

16. Bartel DP. MicroRNAs: genomics, biogenesis, mechanism, and function. Cell. 2004;116(2):281-97. https://doi.org/10.1016/S0092-8674(04)00045-5.

17. Xie F, Jones DC, Wang $Q$, Sun RR, Zhang B. Small RNA sequencing identifies miRNA roles in ovule and fibre development. Plant Biotechnol J. 2015;13(3): 355-69. https://doi.org/10.1111/pbi.12296.

18. Breakfield NW, Corcoran DL, Petricka JJ, Shen J, Sae-Seaw J, Rubio-Somoza I, et al. High-resolution experimental and computational profiling of tissue specific known and novel miRNAs in Arabidopsis. Genome Res. 2012;22(1): 163-76. https://doi.org/10.1101/gr.123547.111.

19. Wang J. Regulation of flowering time by the miR156-mediated age pathway. J Exp Bot. 2014;65(17):4723-30. https://doi.org/10.1093/jxb/eru246.

20. Chen X. A microRNA as a translational repressor of APETALA2 in Arabidopsis flower development. Science. 2004;303(5666):2022-5. https://doi.org/10.112 6/science.1088060

21. Navarro L, Dunoyer P, Jay F, Arnold B, Dharmasiri N, Estelle M, et al. A plant miRNA contributes to antibacterial resistance by repressing auxin signaling. Science. 2006:312(5772):436-9. https://doi.org/10.1126/science.1126088.

22. Sunkar R, Zhu J. Novel and stress-regulated micro-RNAs and other small RNAs from Arabidopsis. Plant Cell. 2004;16(8):2001-19. https://doi.org/10.11 05/tpc. 104.022830

23. Wang Z, Xue W, Dong C, Jin L, Bian S, Wang C, et al. A comparative miRNAome analysis reveals seven fiber initiation-related and 36 novel miRNAs in developing cotton mvules. Mol Plant. 2012;5(4):889-900. https:// doi.org $/ 10.1093 / \mathrm{mp} / \mathrm{ssr} 094$

24. Wang Y, Liu W, Wang $X$, Yang R, Wu Z, Wang H, et al. MiR156 regulates anthocyanin biosynthesis through SPL targets and other microRNAs in poplar. Horticult Res. 2020;7(1):6786-7.

25. Ye B, Zhang K, Wang J. The role of miR156 in rejuvenation in Arabidopsis thaliana. J Integr Plant Biol. 2020;62(05):550-5. https://doi.org/10.1111/ jipb.12855.

26. Teotia S, Tang G. To bloom or not to bloom: role of microRNAs in plant flowering. Mol Plant. 2015;8(3):359-77. https://doi.org/10.1016/j.molp.2 014.12.018.

27. Munusamy P, Zolotarov Y, Meteignier LV, Moffett P, Strömvik MV. novo computational identification of stress-related sequencemotifs and microRNA target sites in untranslated regions of a plant translatome. Sci Rep. 2017;7(1):43861

28. Wu G, Mee YP, Susan RC, Wang J, Detlef W, Poethig RS. The sequential action of miR156 and miR172 regulates developmental timing in Arabidopsis. Cell. 2009;138(4):750-9. https://doi.org/10.1016/j. cell.2009.06.031.

29. Yu N, Cai W, Wang S, Shan C, Wang L, Chen X. Temporal control of trichome distribution by MicroRNA156-targeted SPL genes in Arabidopsis thaliana. Plant Cell. 2010;22(7):2322-35. https://doi.org/10.1105/tpc.109.072579.

30. Xing S, Salinas M, Hohmann S, Berndtgen R, Huijser P. miR156-targeted and nontargeted SBP-Box transcription factors act in concert to secure male fertility in Arabidopsis. Plant Cell. 2010;22:3935-50.

31. Bergonzi S, Albani MC, Themaat EV, Wang R, Schneeberger K, Moerland PD, et al. Mechanisms of age-dependent response to winter temperature in perennial flowering of Arabis alpine. Science. 2013;340(6136):1094-7. https:// doi.org/10.1126/science.1234116.

32. Wang JW, Czech B, Weigel D. miR156-regulated SPL transcription factors define an endogenous flowering pathway in Arabidopsis thaliana. Cell. 2009; 138(4):738-49. https://doi.org/10.1016/j.cell.2009.06.014.

33. Wang ZS, Wang Y, Kohalmi SE, Amyot L, Hannoufa A. SQUAMOSA promoter binding PROTEIN-LIKE 2 controls floral organ development and plant fertility by activating ASYMMETRIC LEAVES 2 in Arabidopsis thaliana. Plant Mol Biol. 2016;9(7):1-14

34. Kim JJ, Lee JH, Kim W, Jung HS, Huijser P, Ahn JH. The miR156-SPL3 module regulates ambient temperature-responsive flowering via FT in Arabidopsis thaliana. Plant Physiol. 2012;159(1):461-78. https://doi.org/10.1104/pp.111.1 92369.

35. Jung JH, Seo PJ, Kang SK, Park CM. miR172 signals are incorporated into the miR156 signaling pathway at the SPL3/4/5 genes in Arabidopsis developmental transitions. Plant Mol Biol. 2011;76(1/2):35-45. https://doi org/10.1007/s11103-011-9759-z.

36. Unte US, Sorensen AM, Pesaresi P. SPL8, an SBP-box gene that affects pollen sac development in Arabidopsis. Plant Cell. 2003;15(4):1009-19. https://doi. org/10.1105/tpc.010678.
37. Hyun YB, Richter R, Vincent C, Martinez-Gallegos R, Porri A, Coupland G. Multi-layered regulation of SPL15 and cooperation with SOC1 integrate endogenous flowering pathways at the Arabidopsis shoot meristem. Dev Cell. 2016;37(3):254-66. https://doi.org/10.1016/j.devcel.2016.04.001.

38. Xing S, Salinas M, Molina AG. SPL8 and miR156-targeted SPL genes redundantly regulate Arabidopsis gynoecium differential patterning. Plant J. 2013;75(4):566-77. https://doi.org/10.1111/tpj.12221.

39. Zhang Y, Schwarz S, Saedler H, Huijser P. SPL8, a local regulator in a subset of gibberellin-mediated developmental processes in Arabidopsis. Plant Mol Biol. 2007;63(3):429-39. https://doi.org/10.1007/s11103-006-9099-6.

40. Yuan Z, Fang Y, Zhang T, Fei Z, Han F, Liu C, et al. The pomegranate (Punica granatum $\mathrm{L}$.) genome provides insights into fruit quality and ovule developmental biology. Plant Biotechnol J. 2018;16(7):1363-74. https://doi. org/10.1111/pbi.12875.

41. Yamaguchi A, Wu MF, Yang L, Wu G, Poethig RS, Wagner D. The microRNAregulated SBP-box transcription factor SPL3 is a direct transcriptional activator of LEAFY, FRUITFULL, and APETALA1. Dev Cell. 2009;17(2):268-78. https://doi.org/10.1016/j.devcel.2009.06.007.

42. Guo A, Zhu Q, Gu X, Ge S, Yang J, Luo J. Genome-wide identification and evolutionary analysis of the plant specific SBP-box transcription factor family. Gene. 2008;418(1):1-8. https://doi.org/10.1016/j.gene.2008.03.016.

43. Xiong J, Zheng D, Zhu H, Chen J, Na R, Cheng Z. Genome-wide identification and expression analysis of the SPL gene family in woodland strawberry Fragaria vesca. Gene. 2018;61(9):675-83.

44. Niu Y, Yang J, Yin G, Li R, Zou W. Genome-wide characterization of the SPL gene family involved in the age development of Jatropha curcas. BMC Genomics. 2020;21(2):585-96.

45. Shen H, Chou K. Cell-PLoc 2.0: an improved package of web-servers for predicting subcellular localization of proteins in various organisms. Nat Sci. 2010:2(10):1090-103.

46. Thomson B, Wellmer F. Molecular regulation of flower development. Curr Top Dev Biol. 2019;131:185-210. https://doi.org/10.1016/bs.ctdb.2018.11.007.

47. Zhang D, Han Z, Li J, Qin H, Zhou L, Wang Y, et al. Genome-wide analysis of the SBP-box gene family transcription factors and their responses to abiotic stresses in tea (Camellia sinensis ). Genomics. 2020;112(3):2194-202. https:// doi.org/10.1016/j.ygeno.2019.12.015.

48. Alim A, Alia KB, Atif RM. Genome-wide identification and comparative analysis of squamosa-promoter binding proteins(Sbp) transcription factor family in Gossypium raimondii and Arabidopsis thaliana. Pak J Bot. 2017;49:1113-26.

49. Zhang S, Ling L. Diversification of SQUAMOSA promoter binding proteinlike (SPL) genes by changes of miR156/529 binding sites in land plants. Plant Gene. 2018;14:55-63. https://doi.org/10.1016/j.plgene.2018.04.007.

50. Schwarz S, Grande AV, Bujdoso N, Saedler H, Huijser P. The microRNA regulated SBP-box genes SPL9 and SPL15 control shoot maturation in Arabidopsis. Plant Mol Biol. 2008;67(1-2):183-95. https://doi.org/10.1007/s111 03-008-9310-z.

51. Cai Y, Lu X, Zhu L. Preliminary research on flower bud differentiation of pink pomegranate. Acta Horticult Sin. 1993;20(1):23-6.

52. Wetzstein HY, Ravid N, Wilkins E, Adriana PM. A morphological and histological characterization of bisexual and male flower types in pomegranate. J Am Soc Hortic Sci. 2011;136(2):83-92. https://doi.org/10.212 73/JASHS.136.2.83

53. Chen LN, Zhang J, Li HX, Niu J, Xue H, Liu BB, et al. Transcriptomic analysis reveals candidate genes for female sterility in pomegranate flowers. Front Plant Sci. 2017:8:1430. https://doi.org/10.3389/fpls.2017.01430.

54. Chen L. Exploring regulatory genes of pomegranate (Punica granatum) female sterility based on transcriptome analysis. 3rd ed. Beijing: Chinese Academy of Agricultural Sciences; 2017.

55. Yamaguchi A, Wu M, Yang L, Wu G, Poethig RS, Wagner D. The MicroRNAregulated SBP-box transcription factor SPL3 is a direct upstream activator of LEAFY, FRUITFULL, and APETALA1. Dev Cell. 2009;17(2009):268-78. https:// doi.org/10.1016/j.devcel.2009.06.007.

56. Jung JH, Ju Y, Seo PJ. The SOC1-SPL module integrates photoperiod and gibberellic acid signals to control flowering time in Arabidopsis. Plant J. 2012;9:577-88.

57. Chuck G, Whipple C, Jackson D, Hake S. The maize SBP-box transcription factor encoded by tasselsheath 4 regulates bract development and the establishment of meristem boundaries. Development. 2010;137(8):1243-50. https://doi.org/10.1242/dev.048348.

58. Preston JC, Hileman LC. SQUAMOSA-PROMOTE RBINDING PROTEIN 1 initiates flowering in Antirrhinum majus through the activation of meristem 
identity genes. Plant J. 2010;62(4):704-12. https://doi.org/10.1111/j.1365-313 X.2010.04184.X.

59. Jin J, Tian F, Yang D, Meng Y, Kong L, Luo JC, et al. PlantTFDB 4.0: toward a central hub for transcription factors and regulatory interactions in plants. Nucleic Acids Res. 2017;45(D1):D1040-5. https://doi.org/10.1093/nar/gkw982.

60. Marchler-Bauer A, Bo Y, Han L, He J, Lanczycki CJ, Lu S, et al. CDD/SPARCLE: functional classification of proteins via subfamily domain architectures. Nucleic Acids Res. 2017;45(D1):D200-3. https://doi.org/10.1093/nar/gkw1129.

61. Letunic I. Bork peer. 20 years of the SMART protein domain annotation resource. Nucleic Acids Res. 2018;46(D1):D493-6. https://doi.org/10.1093/na r/gkx922.

62. Li S, Xu X, Chen X, Chen X, Li H, Lin Y, et al. Cloning and analysis of expression characteristics of CK2 in Dimocarpus longan Lour. J Fruit Sci. 2019;36(12):1638-47.

63. Kumar S, Stecher G, Tamura K. MEGA7: molecular evolutionary genetics analysis version 7.0 for bigger datasets. Mol Biol Evol. 2016;33(7):1870-4. https://doi.org/10.1093/molbev/msw054.

64. Balakrishnan S, Gao S, Lercher MJ, Hu S, Chen W. Evolview v3: a webserver for visualization, annotation, and management of phylogenetic trees. Nucleic Acids Res. 2019;47:W270-5.

65. Bailey TL, Johnson J, Grant CE, Noble-William S. The MEME suite. Nucleic Acids Res. 2015;43(W1):W39-49. https://doi.org/10.1093/nar/gkv416.

66. Guo A, Zhu Q, Chen X, Luo J. GSDS: gene structure display system. Inheritance. 2007;08:1023-6.

67. Magali L, Patrice D, Gert T, Kathleen M, Yves, Yves VP, et al. PlantCARE, a database of plant cis-acting regulatory elements and a portal to tools for in silico analysis of promoter sequences. Nucleic Acids Res. 2020;30:325-7.

68. Dai X, Zhuang Z, Zhao P. psRNATarget: a plant small RNA target analysis server (2017 release). Nucleic Acids Res. 2018;46(W1):W49-54. https://doi. org/10.1093/nar/gky316.

69. Chen S, Zhou Y, Chen Y, Gu J. fastp: an ultra-fast all-in-one FASTQ preprocessor. Bioinformatics. 2018;34(17):i884-90. https://doi.org/10.1093/ bioinformatics/bty560.

70. Chen L, Niu W, Liu B, Jing D, Luo X, Li G, et al. Effects of foliar application of different plant growth regulators on fruit setting rate and quality in pomegranate. J Fruit Sci. 2020;37(02):244-53.

71. Chen L, Li H, Niu J. Study on the growth characteristics and morphological differences of fertile and abortive flowers of Tunisia soft-seed pomegranate. J Fruit Trees. 2017:34:80-7.

72. Sun WX, Shi M, Xue B, Xia Y, Guo Q, Wang S, et al. Cloning, subcellular localization and expression analysis of the EjSPL5 gene in triploid loquat. Acta Horticult Sin. 2020:47(02):220-32.

73. Qi T, Gao M, Yang Y, Li M, Ma F, Ma B. Cloning, expression analysis, and subcellular position of $\mathrm{MdPH} 1$ related to acidity in Malus domestica Borkh. J Plant Sci. 2019;37(06):767-74.

74. Livak KJ, Schmittgen TD. Analysis of relative gene expression data using realtime quantitative PCR and the $2^{-\Delta \Delta C T}$ method. Methods. 2001;25(4):4028. https://doi.org/10.1006/meth.2001.1262

\section{Publisher's Note}

Springer Nature remains neutral with regard to jurisdictional claims in published maps and institutional affiliations.

Ready to submit your research? Choose BMC and benefit from:

- fast, convenient online submission

- thorough peer review by experienced researchers in your field

- rapid publication on acceptance

- support for research data, including large and complex data types

- gold Open Access which fosters wider collaboration and increased citations

- maximum visibility for your research: over $100 \mathrm{M}$ website views per year

At $\mathrm{BMC}$, research is always in progress.

Learn more biomedcentral.com/submissions 